Review

\title{
Current Methods for the Extraction and Analysis of Isothiocyanates and Indoles in Cruciferous Vegetables
}

\author{
Sofia Karanikolopoulou ${ }^{1}$, Panagiota-Kyriaki Revelou ${ }^{1,2, *(\mathbb{D}, \text {, Marinos Xagoraris }}{ }^{2}$ D, Maroula G. Kokotou ${ }^{2}$ \\ and Violetta Constantinou-Kokotou ${ }^{2}$ \\ 1 Department of Food Science and Technology, University of West Attica, Ag. Spyridonos str, Egaleo, \\ 12243 Athens, Greece; ft17035@uniwa.gr \\ 2 Laboratory of Chemistry, Department of Food Science and Human Nutrition, Agricultural University of \\ Athens, 75 Iera Odos, 11855 Athens, Greece; mxagor@aua.gr (M.X.); mkokotou@aua.gr (M.G.K.); \\ vikon@aua.gr (V.C.-K.) \\ * Correspondence: p.revelou@uniwa.gr; Tel.:+30-2105294249
}

check for updates

Citation: Karanikolopoulou, S.; Revelou, P.-K.; Xagoraris, M.;

Kokotou, M.G.;

Constantinou-Kokotou, V. Current Methods for the Extraction and Analysis of Isothiocyanates and Indoles in Cruciferous Vegetables. Analytica 2021, 2, 93-120. https: / / doi.org/10.3390/analytica2040011

Academic Editor: Marcello Locatelli

Received: 14 August 2021

Accepted: 17 September 2021

Published: 24 September 2021

Publisher's Note: MDPI stays neutral with regard to jurisdictional claims in published maps and institutional affiliations.

Copyright: (c) 2021 by the authors. Licensee MDPI, Basel, Switzerland. This article is an open access article distributed under the terms and conditions of the Creative Commons Attribution (CC BY) license (https:// creativecommons.org/licenses/by/ $4.0 /)$.

\begin{abstract}
Cruciferous vegetables are characterized by the presence of sulfur-containing secondary plant metabolites known as glucosinolates (GLS). The consumption of cruciferous vegetables such as broccoli, cabbage, rocket salad, and cauliflower has been related to the prevention of noncommunicable diseases. Their beneficial effects are attributed to the enzymatic degradation products of GLS, e.g., isothiocyanates and indoles. Owing to these properties, there has been a shift in the last few years towards the research of these compounds and a wide range of methods for their extraction and analytical determination have been developed. The aim of this review is to present the sample preparation and extraction procedures of isothiocyanates and indoles from cruciferous vegetables and the analytical methods for their determination. The majority of the references that have been reviewed are from the last decade. Although efforts towards the application of eco-friendly non-conventional extraction methods have been made, the use of conventional solvent extraction is mainly applied. The major analytical techniques employed for the qualitative and quantitative analysis of isothiocyanates and indoles are high-performance liquid chromatography and gas chromatography coupled with or without mass spectrometry detection. Nevertheless, the analytical determination of isothiocyanates presents several problems due to their instability and the absence of chromophores, making the simultaneous determination of isothiocyanates and indoles a challenging task.
\end{abstract}

Keywords: Brassicaceae; sulforaphane; indole-3-carbinol; broccoli; extraction; HPLC; GC; glucosinolates; mass spectrometry

\section{Introduction}

Cruciferous vegetables are members of the Brassicaceae (Cruciferae) family and include vegetables such as broccoli, cauliflower, Chinese cabbage, radish, arugula, cabbage, Brussels sprouts, turnip, watercress, kale, horseradish, garden cress, and wasabi. They are abundant in bioactive compounds, e.g., flavonoids, phenolic acids, carotenoids, terpenoids, phytosterols, and glucosinolates (GLS) [1]. Cruciferous vegetables can be considered functional foods, since their consumption can improve normal body function and can help prevent the development of cancer, cardiovascular disease, diabetes, and chronic inflammation [2-6]. The series of S- and N-secondary metabolites, known as GLS, underpin this beneficial effect. When GLS undergo enzymatic hydrolysis from the endogenous enzyme myrosinase, there is a release of aglucon products, which have a positive impact on human health [7]. Depending on the $\mathrm{pH}$ values, temperature, and other factors, the predominant aglucon products are isothiocyanates (ITCs) and indoles [8]. These compounds also contribute highly to the defense mechanism system of plants against herbivores and pathogens [9-11]. 
One of the most important ITCs is sulforaphane (SFN), which derives from the enzymatic hydrolysis of the glucosinolate glucoraphanin. It is highly effective in activating phase II detoxification enzymes in the human body [12] and suppresses histone deacetylase, and thus has attracted substantial interest [13]. Glucobrassicin is a notable glucosinolate as well, yielding indol-3-ylmethylisothiocyanate upon enzymatic hydrolysis. Nevertheless, this particular isothiocyanate (ITC) lacks stability, so indole-3-carbinol (I3C) is detected instead [14]. The latter may present chemopreventive activity, making it the focus of extensive research in recent times [15-17].

A range of analytical techniques have been devised to determine ITCs and indoles from cruciferous vegetables, owing to the high contribution of these metabolites to human health. Liquid chromatography (LC) is the main technique used to determine ITCs [18-24], while pre-column derivatization has been additionally employed [25]. Moreover, approaches based on gas chromatography (GC) have been reported [24,26-30]. However, in some cases, thermal degradation has been observed [31,32]. These compounds can be also quantified via Liquid Chromatography-Mass Spectrometry (LC-MS) methods, not only in Brassica [33,34] or broccoli products [35], but also in honey [36] and body fluids [37-41]. High Resolution Mass Spectrometry (HRMS) offers additional sensitivity, allowing a more precise and simultaneous determination of ITCs and indoles [42,43]. Moreover, total ITCs have been quantified through UV spectrophotometry [44] and infrared Fourier-Transform spectroscopy [45].

The aim of this review is to provide an overview of the methods that have been employed in the last decade for the conventional and non-conventional extraction of ITCs and indoles from cruciferous vegetables, as well as to present the analytical methods and techniques that have been developed for this purpose. The literature research was performed from March to July 2021, using databases such as Web of Science, Scopus, PubMed, and JSTOR by keywords such as cruciferous vegetables, Brassicaceae, analysis, extraction, and determination.

\section{Cruciferous Vegetables}

Cruciferous vegetables are members of the order Brassicales and the Brassicaceae botanical family (also called Cruciferae). The latter encompasses nearly 400 genera (e.g., Brassica, Raphanus, Armoracia, Nasturtium, Wasabia, Alyssum, Arabidopsis, etc.), and over 4000 species [46,47]. The majority of Brassicaceae species are not comestible, but there are several members of the family such as Brassica oleracea and Brassica rapa that are edible and economically significant (Table 1).

Table 1. Edible Brassicaceae crops.

\begin{tabular}{ccc}
\hline Common Name & Genus & Species and Cultivar \\
\hline Cauliflower & & B. oleracea (L.) var. botrytis \\
Cabbage & & B. oleracea (L.) var. capitata \\
Brussels sprouts & & B. oleracea (L.) var. gemmifera \\
Broccoli & B. oleracea $($ L.) var. italica \\
Savoy cabbage & Brassica & B. oleracea (L.) var. sabauda \\
Kale & & B. oleracea (L.)var. acephala \\
Bok choy & B. rapa var. (L.) chinensis \\
Turnip & B. rapa (L.) var. rapa \\
White mustard & & B. alba (L.) Rabenh. \\
Indian mustard & B. juncea (L.) Czernj \\
Radish & Raphanus & R. raphanistrum subsp. Sativus (L.) Domin \\
Horseradish & Armoracia & A.rusticana $($ L.) \\
Watercress & Nasturtium & N.officinale W.T.Aiton \\
Garden cress & Lepidium & L.sativum (L.) \\
Rocket & Eruca & E. sativa Mill. \\
Wasabi & Wasabia & W.japonica (L.) \\
\hline
\end{tabular}


According to recent epidemiological research, certain types of cancer are less likely to develop when the dietary intake of cruciferous vegetables is high, providing support for the classification of these vegetables as functional foods. [48-52]. Consequently, the market has been inundated with dietary supplements that contain different extracts or compounds derived from cruciferous vegetables. GLS, and the products of their enzymatic hydrolysis are the primary source of the positive health effects of cruciferous vegetables [53], while polyphenols and triterpenes are beneficial to health as well $[54,55]$. The action against cancer, oxidants, inflammation, and cardiac disease that has been attributed to cruciferous vegetables can be also attributed to the synergistic activity of the above-mentioned phytochemicals [55-57].

\section{The Chemistry of Glucosinolates, Isothiocyanates, and Indoles}

Isothiocyanates and indoles are naturally occurring molecules that are produced from the enzymatic hydrolysis of GLS (Figure 1) performed by the enzyme $\beta$-thioglucoside glucohydrolase, EC 3.2.3.1 (myrosinase) [58,59]. Phytoalexins, phytoanticipins, and phytohormones also contain indole rings [14,60-62]. Glucosinolates display chemical stability and are found in members of the order Brassicales and the genus Drypetes (order Malpighiales, family Putranjivaceae) $[59,63]$. They are organic anions soluble in water that contain $\beta$-Dthioglucose and sulfonated oxime moieties. Three types of GLS are classified according to the amino acid precursor structure: (1) aliphatic GLS, derived from methionine, alanine, leucine, valine, or isoleucine, (2) arylaliphatic GLS, derived from phenylalanine or tyrosine, and (3) indole GLS, derived from tryptophan [59]. Cyanogenic glycosides that exist throughout the plant kingdom [64,65], are the basis of the evolution of GLS. The species Carica papaya (order Brassicales, family Caricaceae) is the only plant that contains both cyanogenic glycosides and GLS, and is capable of the concomitant production of cyanogenic glycosides and phenylalanine-derived GLS [66].

During the chewing, cutting, or wounding of cruciferous vegetables, endogenous myrosinase is released and catalyzes the hydrolysis of GLS to glucose with an intermediate lack of stability (Figure 1), which is immediately transformed into different compounds such as isothiocyanates, thiocyanates, nitriles, epithionitriles, and oxazolidine-2-thiones [67]. The ITCs are usually formed when enzymatic hydrolysis occurs at a neutral $\mathrm{pH}$. The generated ITCs are cyclized to oxazolidine-2-thiones if the C-2 of the GLS side chain contains a hydroxyl group. Nitriles are formed in vitro when the $\mathrm{pH}$ level is low and $\mathrm{Fe}^{2+}$ ions are present [68]. The nitrile formation in vivo occurs when protein factors such as the epithiospecifier protein (ESP), thiocyanate-forming protein (TFP), and epithiospecifier modifier protein (EMP) are active $[69,70]$. It is likely that the epithiospecifier modifier (ESM1) interferes with ESP and directs the hydrolysis towards the production of ITCs [71]. Glucosinolates undergo enzymatic hydrolysis to epithionitriles when ESP and TFP are present. Thiocyanates are formed from the hydrolysis of benzyl-, allyl-, and 4-methylsulfinylbutyl GLS [72].

Unlike other GLS hydrolysis products, those with an indole ring are distinct because the ITCs formed at a neutral $\mathrm{pH}$ are unstable and ultimately undergo conversion to indol-methanols, ascorbic acid conjugates, and oligomeric mixtures $[67,70,73]$. When glucobrassicin is hydrolyzed (Figure 2), indole-3-acetonitrile and I3C are formed as a result of the fast reaction of the unstable isothiocyanate intermediate. The formation of indole-3-acetonitrile is promoted by the ESP protein in a way that is comparable to aliphatic GLS. Furthermore, the epithiospecifier modifier 1 gene (ESM1) $[74,75]$ regulates indole-3-acetonitrile generation as well. Compared to indole-3-acetonitrile, I3C displays a greater chemical instability and yields products such as 3,3-diindindolylmethane, indole-3carboxaldehyde, and ascorbigen (Figure 2) [14,73]. 
<smiles>[R]C(=NOS(=O)(=O)[O-])SC1OC(O)C(O)C(O)C1O</smiles>

Glucosinolate
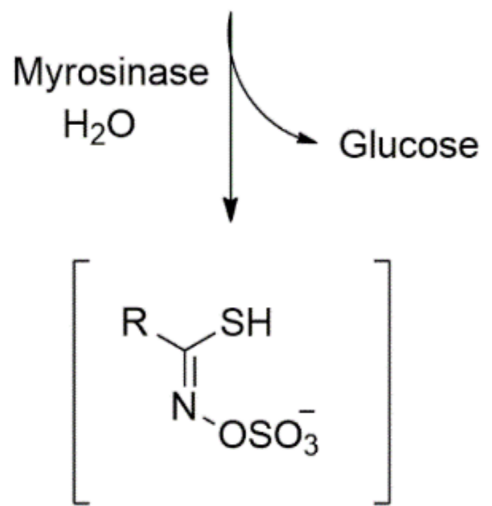

Thioxydroxamate-O-sulfonate

(Unstable intermediate)

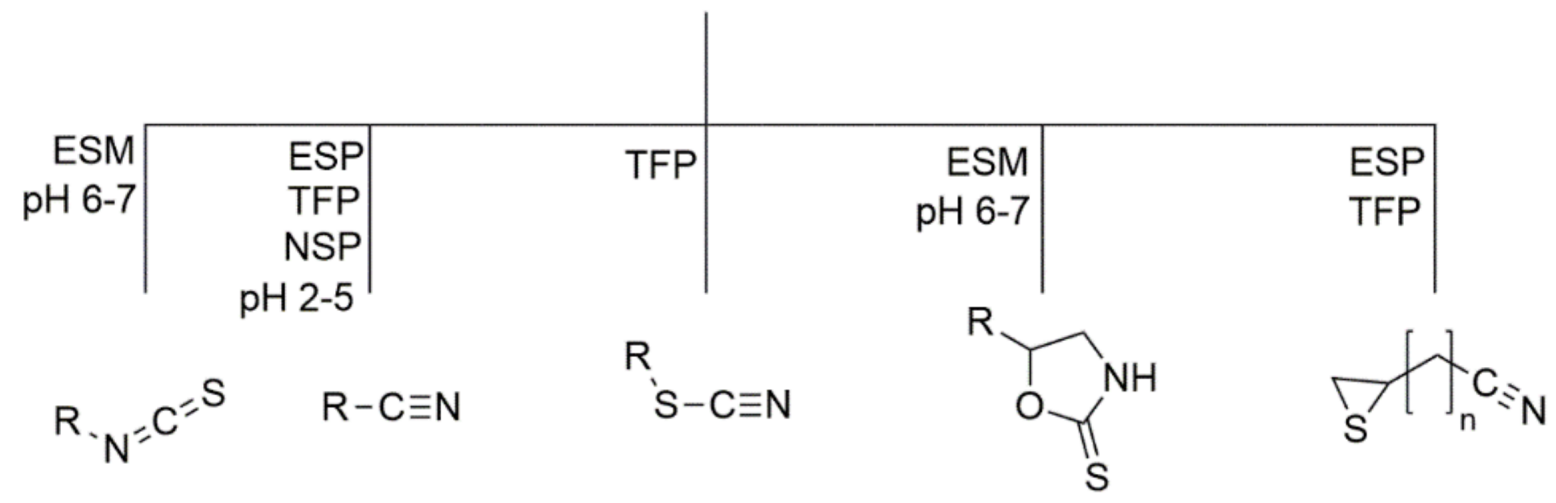

Isothiocyanates Nitriles Thiocyanates Oxazolidine-2-thiones Epithionitriles

Figure 1. Chemical structures of aliphatic glucosinolates and their enzymatic hydrolysis products. ESP, epithiospecifier protein; NSP, nitrile-specifier proteins; TFP, thiocyanate-forming protein; ESM, epithiospecifier modifier protein. 


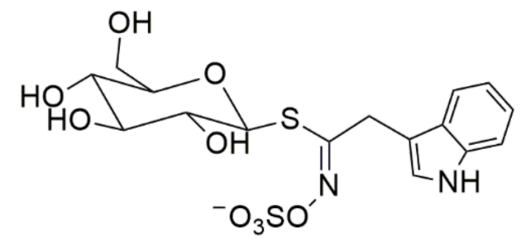

Glucobrassicin

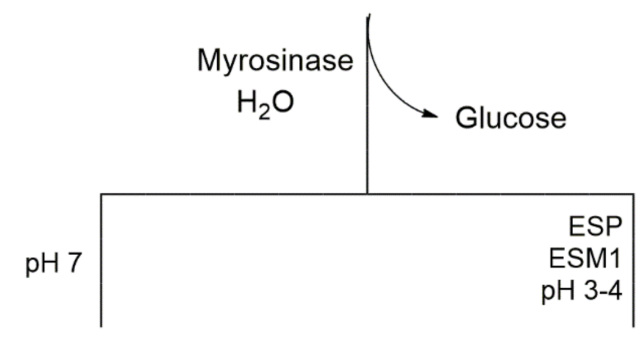<smiles>S=C=NCc1c[nH]c2ccccc12</smiles>

Indol-3-yl-methyl isothiocyanate

(Unstable)<smiles>OCc1c[nH]c2ccccc12</smiles>

Indole-3-carbinol

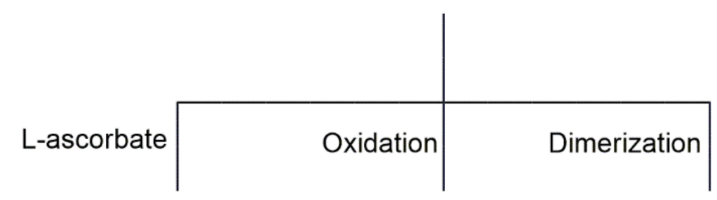<smiles>O=C1O[C@@H]2[C@@H](O)CO[C@H]2[C@](O)(Cc2c[nH]c3ccccc23)[C@@H]1O</smiles>

Ascorbigen<smiles>O=Cc1c[nH]c2ccccc12</smiles>

Indole-3-carboxaldehyde<smiles>c1ccc2c(Cc3c[nH]c4ccccc34)c[nH]c2c1</smiles>

3,3'-Diindolymethane

Figure 2. Chemical structure of glucobrassicin and its enzymatic hydrolysis products. ESP, epithiospecifier protein; ESM1, epithiospecifier modifier 1.

\section{Content of Isothiocyanates and Indoles in Cruciferous Vegetables}

The content of isothiocyanates has been studied mainly in broccoli as it contains the highest concentration of total ITCs compared to other cruciferous vegetables, with the anticancer isothiocyanate SFN predominating. Sulforaphane, contrary to other ITCs, is present in a wide range of cruciferous vegetables [28,31,46,76-78]. The contents of various ITCs and indoles in cruciferous vegetables are summarized in Table 2. 
Table 2. Content of isothiocyanates and indoles in cruciferous vegetables.

\begin{tabular}{|c|c|c|c|c|c|}
\hline Glucosinolate & Products & Structure & Vegetable & Content & References \\
\hline Glucoraphanin & Sulforaphane & & $\begin{array}{l}\text { Broccoli } \\
\text { White cabbage } \\
\text { Red cabbage } \\
\text { Turnip } \\
\text { Cauliflower } \\
\text { Arugula } \\
\text { Radish }\end{array}$ & $\begin{array}{c}0.14-370.3 \mathrm{mg} / 100 \mathrm{~g} \mathrm{FW}^{1} \\
540 \mu \mathrm{g} / \mathrm{g} \mathrm{FW} \\
48 \mu \mathrm{g} / \mathrm{g} \mathrm{FW} \\
60 \mu \mathrm{g} / \mathrm{g} \mathrm{FW} \\
2-190 \mu \mathrm{mol} / 100 \mathrm{~g} \mathrm{FW} \\
5.90 \mu \mathrm{mol} / \mathrm{g} \mathrm{DW}^{2} \\
111.94 \mu \mathrm{g} / \mathrm{g} \mathrm{FW}^{2}\end{array}$ & $\begin{array}{l}{[31,76]} \\
{[28]} \\
{[46]} \\
{[77]} \\
{[78]}\end{array}$ \\
\hline Glucoiberin & Iberin & & $\begin{array}{l}\text { Broccoli } \\
\text { White cabbage } \\
\text { Cauliflower } \\
\text { Arugula }\end{array}$ & $\begin{array}{c}2.40 \mathrm{mg} / \mathrm{g} \mathrm{DW} \\
5-280 \mu \mathrm{mol} / 100 \mathrm{~g} \mathrm{FW} \\
0-330 \mu \mathrm{mol} / 100 \mathrm{~g} \mathrm{FW} \\
1.55 \mu \mathrm{mol} / \mathrm{g} \mathrm{DW}\end{array}$ & $\begin{array}{l}{[79]} \\
{[46]} \\
{[77]}\end{array}$ \\
\hline Glucoreucin & Erucin & & Broccoli seeds & $0.38-1.08 \mathrm{mg} / \mathrm{g}$ & [80] \\
\hline Glucobrassicin & Indole-3-carbinol & & $\begin{array}{l}\text { Broccoli } \\
\text { White cabbage } \\
\text { Cauliflower }\end{array}$ & $\begin{array}{l}24.6 \mu \mathrm{mol} / 100 \mathrm{~g} \mathrm{DW} \\
0.116 \mu \mathrm{mol} / 100 \mathrm{~g} \mathrm{FW} \\
39.5 \mu \mathrm{mol} / 100 \mathrm{~g} \mathrm{DW}\end{array}$ & $\begin{array}{l}{[81]} \\
{[23]} \\
{[81]}\end{array}$ \\
\hline Sinigrin & Allyl-ITC & & $\begin{array}{c}\text { Broccoli } \\
\text { White cabbage } \\
\text { Turnip } \\
\text { Arugula }\end{array}$ & $\begin{array}{c}7.54 \mathrm{mg} / \mathrm{g} \mathrm{DW} \\
4-160 \mu \mathrm{mol} / 100 \mathrm{~g} \mathrm{FW} \\
5 \mathrm{mg} / 100 \mathrm{~g} \mathrm{FW} \\
1-160 \mu \mathrm{mol} / 100 \mathrm{~g} \mathrm{FW}\end{array}$ & $\begin{array}{l}{[79]} \\
{[46]} \\
{[82]} \\
{[46]}\end{array}$ \\
\hline Gluconasturtiin & Phenethyl-ITC & & $\begin{array}{c}\text { Broccoli } \\
\text { Turnip } \\
\text { Watercress }\end{array}$ & $\begin{array}{c}1.93 \mathrm{mg} / \mathrm{g} \mathrm{DW} \\
8 \mathrm{mg} / 100 \mathrm{~g} \mathrm{FW} \\
14-29.3 \mu \mathrm{mol} / \mathrm{g} \mathrm{DW}\end{array}$ & $\begin{array}{l}{[79]} \\
{[82]} \\
{[83]}\end{array}$ \\
\hline Glucobrassicin & Ascorbigen & & $\begin{array}{l}\text { Broccoli } \\
\text { White cabbage } \\
\text { Cauliflower }\end{array}$ & $\begin{array}{c}236 \mu \mathrm{mol} / 100 \mathrm{~g} \mathrm{DW} \\
0.081 \mu \mathrm{mol} / 100 \mathrm{~g} \mathrm{FW} \\
929 \mu \mathrm{mol} / 100 \mathrm{~g} \mathrm{DW}\end{array}$ & $\begin{array}{l}{[81]} \\
{[23]} \\
{[81]}\end{array}$ \\
\hline Glucobrassicin & $\begin{array}{c}\text { Indole-3- } \\
\text { carboxaldehyde }\end{array}$ & & $\begin{array}{c}\text { White cabbage } \\
\text { Cauliflower }\end{array}$ & $\begin{array}{c}1.88 \mathrm{mg} / 100 \mathrm{~g} \mathrm{FW} \\
10 \mu \mathrm{g} / 100 \mathrm{~g} \mathrm{FW}\end{array}$ & [84] \\
\hline Glucobrassicin & $\begin{array}{c}3,3^{\prime}- \\
\text { Diindolylmethane }\end{array}$ & & $\begin{array}{l}\text { Broccoli } \\
\text { White cabbage } \\
\text { Cauliflower }\end{array}$ & $\begin{array}{c}3.1 \mu \mathrm{mol} / 100 \mathrm{~g} \mathrm{DW} \\
0.00235 \mu \mathrm{mol} / 100 \mathrm{~g} \mathrm{FW} \\
3.1 \mu \mathrm{mol} / 100 \mathrm{~g} \mathrm{DW}\end{array}$ & $\begin{array}{l}{[81]} \\
{[23]} \\
{[81]}\end{array}$ \\
\hline
\end{tabular}

${ }^{1} \mathrm{FW}$ : fresh weight; ${ }^{2}$ DW: dry weight.

Broccoli, in addition to SFN, contains a variety of other ITCs and indole derivatives with significant biological activity such as allyl-ITC, phenethyl-ITC, iberin [79], erucin [80], ascorbigen, I3C, and 3,3'-diindolylmethane [81]. White cabbage and cauliflower are also important sources of ITCs and indoles such as SFN [28,46], iberin [46], allyl-ITC [46,82], ascorbigen, I3C, and 3,3'-diindolylmethane [23,81]. Turnips contain SFN [28] and phenethylITC [82], while arugula (salad rocket) contains SFN and iberin [77]. Phenethyl-ITC is mainly present in watercress [83], while indole-3-carboxaldehyde has been detected in white cabbage and cauliflower [84].

The content of these compounds is indicative as it is influenced by numerous factors including variety, geography, season, and environmental factors, e.g., the infestation of pathogenic microorganisms, soil fertility, and plant growth regulators [85]. In addition, another important factor to note is the sample preparation and extraction conditions. The enzymatic hydrolysis of GLS must be complete and under appropriate conditions conducive to the production of ITCs over the corresponding nitriles. This explains the 
discrepancies between studies regarding the SFN concentration in broccoli, which has been reported to be in the range of $0.14-370.3 \mathrm{mg} / 100 \mathrm{~g}$ fresh weight (Table 2). [84]

\section{Extraction Procedures}

\subsection{Conventional Extraction}

The optimum extraction conditions must be chosen carefully, given that ITCs and indoles are the products of GLS enzymatic hydrolysis. Glucosinolates have to undergo complete hydrolysis to ITCs rather than other compounds (e.g., nitriles) that exhibit a potent genotoxic risk [86]. In conventional extraction (Table 3), fresh or lyophilized samples are subjected to homogenization in a blender, with the addition of water or a buffer solution [25,87-89]. For Brassicaceae seeds, $\mathrm{n}$-hexane is usually employed as the solvent for defatting purposes before the addition of water or a buffer. In this step, sonication for several minutes may be used [90]. To complete the hydrolysis of GLS, the mix is then allowed to autolyze at an ambient temperature or is subjected to incubation in a water bath at $35-45^{\circ} \mathrm{C}$ for $1-4 \mathrm{~h}$. A buffer solution is typically added instead of deionized water because it affords a constant neutral $\mathrm{pH}$ that is required to ensure that GLS hydrolysis yields ITCs $[25,87,91,92]$. Nevertheless, it has been argued that for SFN, water with a $\mathrm{pH}$ of 3-6 should be added to attain the maximum amount of this compound when glucoraphanin is hydrolyzed [19,93]. Once hydrolysis is completed, a suitable solvent is used for the extraction of ITCs and indoles. At this step, derivatization may be also applied $[25,87,94]$. Table 3 lists the organic solvents necessary for standard ITC and indole extraction, with the chlorinated solvents dichloromethane $\left(\mathrm{CH}_{2} \mathrm{Cl}_{2}\right)[42,43,78,93,95-97]$ and chloroform $\left(\mathrm{CHCl}_{3}\right)$ [90] being preferred. Nevertheless, other solvents have been also proposed such as ethyl acetate (AcOEt) [98] acetone [96,99], and methyl t-butyl ether (MTBE) $[19,96]$. The general procedure is briefly described in Figure 3.

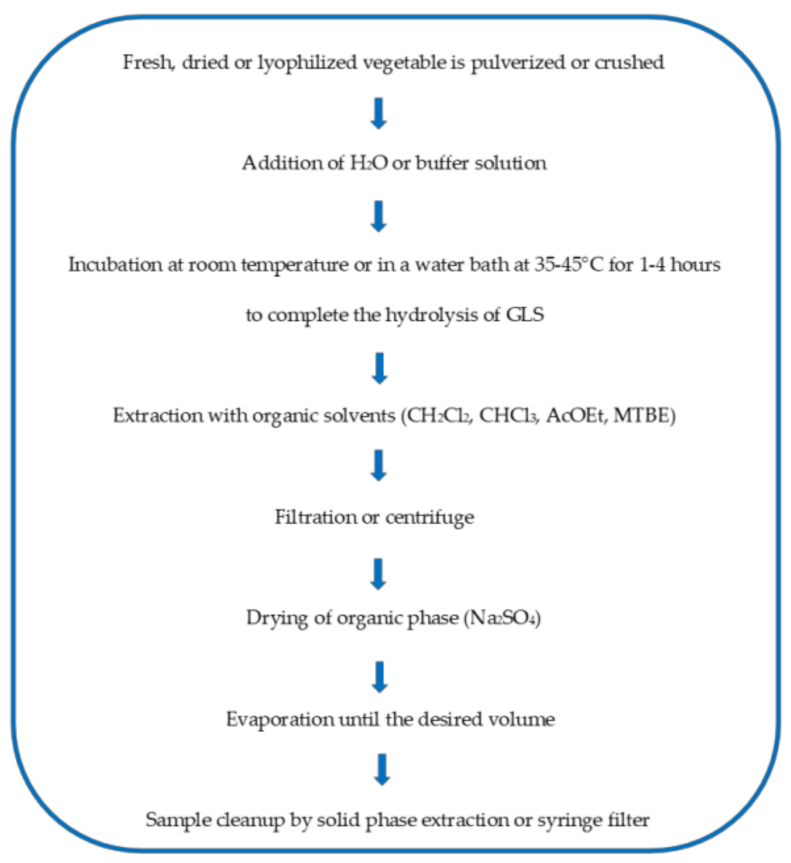

Figure 3. General procedure for the sample preparation and extraction of the hydrolysis products of glucosinolates from cruciferous vegetables.

In the case of analysis using gas chromatography with a flame ionization detector (GC-FID) or a mass spectrometer (GC-MS), $\mathrm{CH}_{2} \mathrm{Cl}_{2}$ is mainly used for the extraction of the hydrolysis products of GLS (Table 4) [100-106]. Additionally, Clevenger hydrodistillation has been also employed to extract the essential oil of cruciferous vegetables in which a great variety of ITCs, nitriles, epithionitriles, and oxazolidine-2-thiones have been identified $[107,108]$. 
Table 3. Sample preparation, conventional extraction, and determination of isothiocyanates and indoles using liquid chromatography/mass spectrometry.

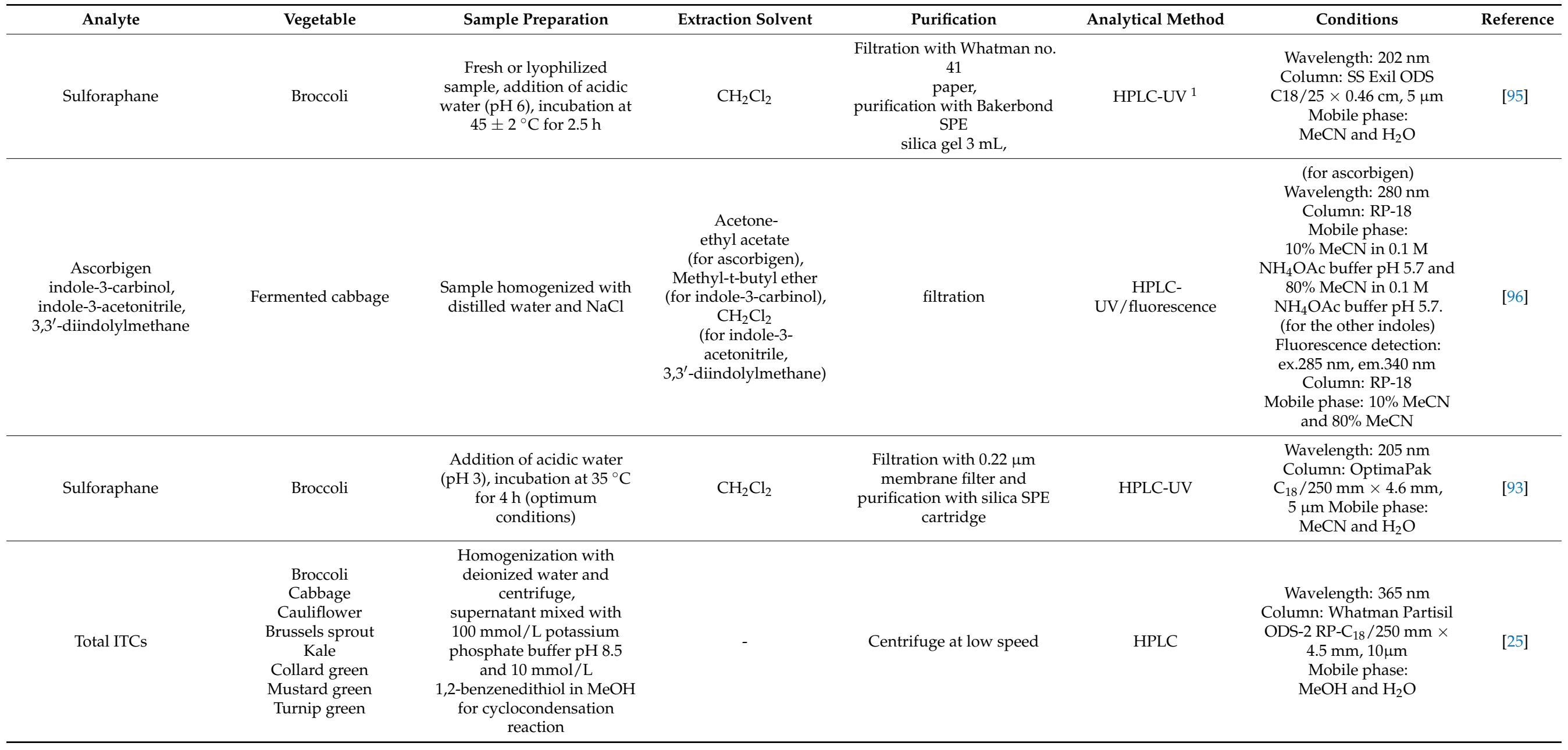


Table 3. Cont

\begin{tabular}{|c|c|c|c|c|c|c|c|}
\hline Analyte & Vegetable & Sample Preparation & Extraction Solvent & Purification & Analytical Method & Conditions & Reference \\
\hline Sulforaphane & $\begin{array}{l}\text { Broccoli florets, stems, } \\
\text { and leaves }\end{array}$ & $\begin{array}{c}\text { Dried sample at } 40^{\circ} \mathrm{C}, \\
\text { addition of acidic water } \mathrm{pH} \\
5.0 \text { or } 6.0 \text { using } 0.1 \mathrm{M} \\
\text { hydrochloric acid, } \\
\text { incubation at } 35-45^{\circ} \mathrm{C} \text { for } \\
1-4 \mathrm{~h}\end{array}$ & Methyl-t-butyl ether & $\begin{array}{l}\text { Filtration with Albet } 140 \\
\text { paper and purification with } \\
\text { SPE with silica } \\
\text { cartridges }\end{array}$ & HPLC-DAD $^{2}$ & $\begin{array}{l}\text { Wavelength: } 196 \mathrm{~nm} \\
\text { Column: Synergi } \\
\text { Hydro-RP } \mathrm{C}_{18} / 150 \mathrm{~mm} \\
\times 4.6 \mathrm{~mm}, 4 \mu \mathrm{m} \\
\text { Mobile phase: } 20 \mathrm{mM} \\
\mathrm{NH}_{4} \mathrm{HCO}_{2} \text { in } \mathrm{H}_{2} \mathrm{O} \text { and } \\
\mathrm{MeCN}(55: 45, v / v)\end{array}$ & [19] \\
\hline $\begin{array}{l}\text { Sulforaphane, } \\
\text { sulforaphene }\end{array}$ & $\begin{array}{l}\text { Raphanus sativus L. var. } \\
\text { caudatus Alef }\end{array}$ & $\begin{array}{l}\text { Homogenization with } \\
\text { deionized water for } 30 \mathrm{~min} \\
\text { and autolyze at r.t. for } 2 \mathrm{~h} \text {. }\end{array}$ & $\mathrm{CH}_{2} \mathrm{Cl}_{2}$ & - & HPLC-DAD & $\begin{array}{c}\text { Wavelength: } 254 \mathrm{~nm} \\
\text { Column: HiQsil } \\
\text { RP- } \mathrm{C}_{18} / 250 \mathrm{~mm} \times 4.6 \mathrm{~mm} \\
5 \mu \mathrm{m} \\
\text { Mobile phase: THF and } \\
\mathrm{H}_{2} \mathrm{O}\end{array}$ & [78] \\
\hline Total ITCs & \multirow{2}{*}{$\begin{array}{c}\text { Fresh and cooked } \\
\text { green cauliflower, } \\
\text { purple cauliflower, } \\
\text { rutabaga }\end{array}$} & $\begin{array}{l}\text { Lyophilized samples mixed } \\
\text { with } 0.01 \mathrm{M} \text { sodium } \\
\text { phosphate buffer } \mathrm{pH} 7.4 \\
\text { incubation at } \\
37^{\circ} \mathrm{C} \text { for } 3 \mathrm{~h} \text {, centrifuge and } \\
\text { purification by SPE. } \\
\text { Retained ITCs submitted to } \\
\text { a cyclocondensation } \\
\text { reaction }\end{array}$ & - & \multirow{2}{*}{$\begin{array}{l}\text { Supernatant passed through } \\
\text { Bakerbond SPE C18 } 500 \mathrm{mg} \\
\text { cartridge. Elution of ITCs } \\
\text { and indoles with } \mathrm{MeOH}\end{array}$} & HPLC-DAD & $\begin{array}{c}\text { Wavelength: } 365 \mathrm{~nm} \\
\text { Zorbax Eclipse } \\
\text { XDB-C } 8 / 150 \mathrm{~mm} \times 4.6 \mathrm{~mm}, \\
3.5 \mu \mathrm{m} \\
\text { Mobile phase: } 1 \%(v / v) \\
\text { formic acid in } \mathrm{H}_{2} \mathrm{O} \text { and } \\
\text { MeOH }\end{array}$ & \multirow[t]{2}{*}{ [87] } \\
\hline $\begin{array}{l}\text { Indole-3-carbinol } \\
\text { Indole-3-acetic acid } \\
\text { Indole-3-acetonitrile } \\
\text { Diindolylmethane } \\
\text { Total indoles }\end{array}$ & & $\begin{array}{c}\text { Lyophilized samples mixed } \\
\text { with } 0.01 \mathrm{M} \text { sodium } \\
\text { phosphate buffer } \mathrm{pH} 7.4, \\
\text { incubation at } \\
37^{\circ} \mathrm{C} \text { for } 3 \mathrm{~h} \text { and centrifuge }\end{array}$ & - & & HPLC-DAD-FLD ${ }^{3}$ & $\begin{array}{c}\text { Fluorescence detection: } \\
\text { ex.280 nm, em. } 360 \mathrm{~nm} \\
\text { Column: Zorbax Eclipse } \\
\text { XDB- } \mathrm{C}_{8} / 150 \mathrm{~mm} \times 4.6 \mathrm{~mm} \text {, } \\
3.5 \mu \mathrm{m} \\
\text { Mobile phase: } \\
\text { MeCN and } \mathrm{H}_{2} \mathrm{O}\end{array}$ & \\
\hline Indole-3-carbinol & $\begin{array}{l}\text { Broccoli, } \\
\text { cabbage }\end{array}$ & $\begin{array}{l}\text { Freeze-dried samples } \\
\text { homogenized with sodium } \\
\text { dihydrogen phosphate and } \\
\text { citric acid buffer for } 1.5 \mathrm{~h}\end{array}$ & Ethyl acetate & $\begin{array}{l}\text { Centrifuge for } 10 \mathrm{~min} \text { at } \\
5500 \times g \text { and filtration with } \\
\text { Agela No. } 0.22-\mu \mathrm{m}(\mathrm{D}) \\
\text { nylon filter paper }\end{array}$ & HPLC-UV & $\begin{array}{c}\text { Wavelength: } 279 \mathrm{~nm} \\
\text { Column: } \mathrm{C}_{18} / 250 \times 4.6 \mathrm{~mm} \text {, } \\
5 \mu \mathrm{m} \\
\text { Mobile phase: } \\
\mathrm{H}_{2} \mathrm{O} \text { and MeCN }\end{array}$ & [92] \\
\hline
\end{tabular}


Table 3. Cont

\begin{tabular}{|c|c|c|c|c|c|c|c|}
\hline Analyte & Vegetable & Sample Preparation & Extraction Solvent & Purification & Analytical Method & Conditions & Reference \\
\hline Alyll-ITC & Mustard & $\begin{array}{l}\text { Samples homogenized with } \\
\text { LC-grade water and ACN at } \\
450 \mathrm{rpm} \text { for } 10 \mathrm{~min} \text { and } \\
\text { sonicated for } 30 \mathrm{~min}\end{array}$ & - & $\begin{array}{l}\text { Centrifuge for } 10 \mathrm{~min} \text { at } \\
\quad 1300 \times g \text { at } 7^{\circ} \mathrm{C} \text { and } \\
\text { filtration with Phenomenex } \\
\text { RC } 0.45 \mu \mathrm{m} \text { membrane filter }\end{array}$ & HPLC-PDA-UV ${ }^{4}$ & $\begin{array}{c}\text { Wavelength: } 242 \mathrm{~nm} \\
\text { Column: ReproSil-Pur } 120 \\
\mathrm{C}_{18}-\mathrm{AQ} / 250 \times 4.6 \mathrm{~mm}, 5 \\
\mu \mathrm{m} \text { Mobile phase: } \mathrm{H}_{2} \mathrm{O} \text { with } \\
0.5 \% \text { formic acid and MeCN } \\
\text { with } 0.5 \% \text { formic acid }\end{array}$ & [88] \\
\hline Sulforaphane & Broccoli & Fresh sample homogenized & $\mathrm{CH}_{2} \mathrm{Cl}_{2}$ & Filtration & HPLC-DAD & $\begin{array}{c}\text { Wavelength: } 254 \mathrm{~nm} \\
\text { Column: Agilent } \mathrm{C}_{18} / 250 \times \\
4.6 \mathrm{~mm}, 5 \mu \mathrm{m} \\
\text { Mobile phase: } \\
\mathrm{H}_{2} \mathrm{O} \text { and MeCN }\end{array}$ & [97] \\
\hline $\begin{array}{c}\text { Ascorbigen, } \\
\text { indole-3-carbinol, } \\
\text { indole-3-acetonitrile, } \\
\text { 3,3'-diindolylmethane }\end{array}$ & Fermented cabbage & $\begin{array}{l}\text { Sample homogenized with } \\
\text { water }\end{array}$ & $\begin{array}{c}\text { Acetone } \\
\text { (for ascorbigen), } \\
\text { Methyl-t-butyl ether } \\
\text { (for indole-3-carbinol), } \\
\mathrm{CH}_{2} \mathrm{Cl}_{2} \\
\text { (for indole-3- } \\
\text { acetonitrile, } \\
\text { 3,3'-diindolylmethane) }\end{array}$ & $\begin{array}{l}\text { Filtration with PTFE filter } \\
0.22 \mu \mathrm{m} \text { (for ascorbigen, } \\
\text { indole-3-acetonitrile, } \\
\text { 3,3'-diindolylmethane), } \\
\text { filtration with filter paper } \\
\text { Munktell, grade: } 390 \text { (for } \\
\text { indole-3-carbinol) }\end{array}$ & HPLC-UV & $\begin{array}{c}\text { (for ascorbigen) } \\
\text { Wavelength: } 280 \mathrm{~nm} \\
\text { Column: LiChrospher }{ }^{\circledR} 100 \\
\text { RP-18/250 } \times 4 \text { mm, } 5 \mu \mathrm{m} \\
\text { Mobile phase: } \\
10 \% \text { MeCN in } 0.1 \mathrm{M} \\
\mathrm{NH}_{4} \mathrm{OAc} \text { buffer pH } 5.7 \text { and } \\
80 \% \text { MeCN in } 0.1 \mathrm{M} \\
\mathrm{NH}_{4} \mathrm{OAc} \text { buffer pH } 5.7 . \\
\text { (for the other indoles) } \\
\text { Fluorescence detection: } \\
\text { ex.285 nm, em. } 340 \mathrm{~nm} \\
\text { Column: LiChrospher }{ }^{\circledR} 100 \\
\text { RP- } 18 / 250 \times 4 \mathrm{~mm}, \\
5 \mu \mathrm{m} \\
\text { Mobile phase: } 10 \% \mathrm{MeCN} \\
\text { and } 80 \% \mathrm{MeCN}\end{array}$ & [99] \\
\hline Sulforaphane, iberin & Broccolini, kale & $\begin{array}{l}\text { Freeze-dried samples } \\
\text { extracted with MilliQ- } \mathrm{H}_{2} \mathrm{O} \\
\text { for } 24 \mathrm{~h} \text { at room } \\
\text { temperature }\end{array}$ & $\mathrm{H}_{2} \mathrm{O}$ & $\begin{array}{l}\text { Centrifuge for } 5 \mathrm{~min} \text { at } \\
\qquad 17,500 \times g\end{array}$ & $\begin{array}{l}\text { UHPLC-QqQ- } \\
\text { MS/MS }\end{array}$ & $\begin{array}{c}\text { Column: ZORBAX Eclipse } \\
\text { Plus } \mathrm{C}_{18} / 50 \times 2.1 \mathrm{~mm}, \\
1.8 \mu \mathrm{m} \\
\text { Mobile phase: } \\
\mathrm{H}_{2} \mathrm{O} / \text { ammonium acetate } 13 \\
\mathrm{mM}(\mathrm{pH} 4) \text { with acetic acid } \\
\text { 99.99:0.01 } v / v \text { and } \\
\text { acetonitrile/acetic acid; } \\
99.99: 0.1, v / v\end{array}$ & [89] \\
\hline
\end{tabular}


Table 3. Cont.

\begin{tabular}{|c|c|c|c|c|c|c|c|}
\hline Analyte & Vegetable & Sample Preparation & Extraction Solvent & Purification & Analytical Method & Conditions & Reference \\
\hline $\begin{array}{c}\text { 3-(methylsulfinyl)propyl- } \\
\text { ITC, } \\
\text { 4-(methylsulfinyl)butyl- } \\
\text { ITC, } \\
\text { 6-(methylsulfinyl)hexyl- } \\
\text { ITC, } \\
\text { 9-(methylsulfinyl)nonyl- } \\
\text { ITC, } \\
\text { 4-(methylsulfinyl)-3- } \\
\text { Butenyl-ITC, } \\
\text { 3-(methylsulfonyl)propyl- } \\
\text { ITC, } \\
\text { 3-(methylthio)propyl-ITC, } \\
\text { 4-(methylthio)butyl-ITC, } \\
\text { 5-(methylthio)pentyl-ITC, } \\
\text { propyl-ITC, } \\
\text { allyl-ITC, } \\
\text { 3-butenyl-ITC, } \\
\text { 4-pentenyl-ITC, } \\
\text { benzyl-ITC, } \\
\text { phenethyl-ITC }\end{array}$ & $\begin{array}{l}\text { Seeds of Sinapis alba } \\
\text { (yellow mustard) } \\
\text { Brassica napus } \\
\text { Brassica juncea var. rugosa } \\
\text { rugosa (Chinese mustard) }\end{array}$ & $\begin{array}{l}\text { Extraction of GLS with } \\
\text { MeOH at } 65^{\circ} \mathrm{C} \text {. } \\
\text { Extract evaporated, } \\
\text { resolubilized in tert-butanol } \\
\text { and freeze-dried. } \\
\text { Addition of } \\
\text { myrosinase and } \\
\text { derivatization with } \\
\text { N-acetyl-L-cysteine }\end{array}$ & - & - & RP-UHPLC-ESI-MS ${ }^{6}$ & $\begin{array}{c}\text { Column: Acquity } \\
\text { UPLC-BEH shield } \\
\text { RP18/150 mm } \\
\times 2.1 \mathrm{~mm}, 1.7 \mu \mathrm{m} \\
\text { Mobile phase: } 0.1 \%(\mathrm{v} / \mathrm{v}) \\
\text { FA in } \mathrm{H}_{2} \mathrm{O} \text { and } 0.1 \%(v / v) \\
\text { FA in } \mathrm{MeCN}\end{array}$ & [94] \\
\hline $\begin{array}{c}\text { Indole-3-carbinol, } \\
\text { indole-3-carboxaldehyde, } \\
\text { ascorbigen, } \\
\text { indole-3-acetic acid }\end{array}$ & $\begin{array}{l}\text { White cauliflower, } \\
\text { red cabbage, } \\
\text { white cabbage, } \\
\text { green broccoli, } \\
\text { purple broccoli, } \\
\text { radish, } \\
\text { turnip }\end{array}$ & $\begin{array}{l}\text { Lyophilized samples mixed } \\
\text { with distilled water, } \\
\text { incubation at } \\
45 \pm 3^{\circ} \mathrm{C} \text { for } 3 \mathrm{~h}\end{array}$ & $\mathrm{CH}_{2} \mathrm{Cl}_{2}$ & $\begin{array}{c}\text { Filtration with Whatman } \\
\text { filter } \\
\text { paper grade } 1\end{array}$ & LC-Q-TOF-MS ${ }^{8}$ & $\begin{array}{c}\text { Column: Agilent Zorbax } \\
\text { C18/50 } \times 2.1 \mathrm{~mm}, 1.8 \mu \mathrm{m} \\
\text { Mobile phase: } \\
\text { MeOH and } \mathrm{H}_{2} \mathrm{O}\end{array}$ & [43] \\
\hline
\end{tabular}

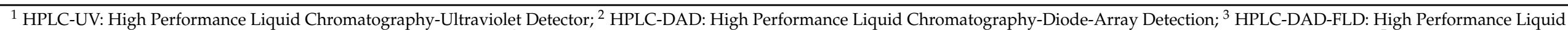

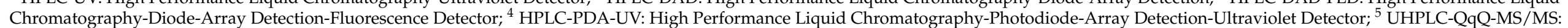

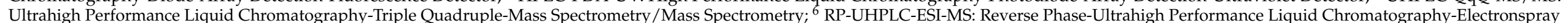

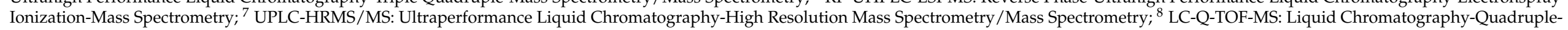
Time of Flight-Mass Spectrometry. 
Table 4. Sample preparation, conventional extraction and determination of isothiocyanates and indoles analysis using gas chromatography/mass spectrometry.

\begin{tabular}{|c|c|c|c|c|c|c|c|}
\hline Analyte & Vegetable & Sample Preparation & Extraction Solvent & Purification & Analytical Method & Conditions & Reference \\
\hline $\begin{array}{l}\text { 4-methylthio-3-butenyl- } \\
\text { ITC }\end{array}$ & $\begin{array}{l}\text { Chinese } \\
\text { radish roots }\end{array}$ & $\begin{array}{l}\text { Samples cut into } 1 \mathrm{~cm} \text { cubic } \\
\text { pieces, blended and ground. } \\
\text { Filtration with two layers of } \\
\text { cotton gauze. Centrifuge of } \\
\text { the filtrate with } \mathrm{CH}_{2} \mathrm{Cl}_{2} \text { at } \\
\quad 3 \times \mathrm{g} \text { for } 1.5 \mathrm{~h} \text { at } 37^{\circ} \mathrm{C}\end{array}$ & $\mathrm{CH}_{2} \mathrm{Cl}_{2}$ & $\begin{array}{l}\text { Centrifuge for } 15 \mathrm{~min} \text { at } \\
\quad 756 \times g \\
\text { Filtration with } 0.45 \mu \mathrm{m} \\
\text { membrane filter }\end{array}$ & GC-FID $^{1}$ & $\begin{array}{c}\text { Column: } \mathrm{HP}-5 / 30 \mathrm{~m} \times \\
0.32 \mathrm{~mm} \times 0.5 \mu \mathrm{m}, \\
\text { splitless mode, } \\
\text { T injector }=250^{\circ} \mathrm{C} \\
\text { T detector }=280^{\circ} \mathrm{C}\end{array}$ & [100] \\
\hline $\begin{array}{l}\text { 4-methylthio-3-butenyl- } \\
\text { ITC }\end{array}$ & Chinese white radish & $\begin{array}{c}\text { Cooked samples were } \\
\text { homogenized and filtered } \\
\text { through two layers of cotton } \\
\text { gauze. Ultrasonic extraction } \\
\text { for } 10 \text { min }\end{array}$ & $\mathrm{CH}_{2} \mathrm{Cl}_{2}$ & $\begin{array}{l}\text { Centrifuge for } 10 \mathrm{~min} \text { at } \\
3000 \mathrm{rpm}\end{array}$ & GC-FID & $\begin{array}{c}\text { Column: HP-5/30 m } \times 0.25 \\
\text { mm } \times 0.25 \mu \mathrm{m}, \\
\text { splitless mode, } \\
\text { T injector }=200{ }^{\circ} \mathrm{C} \\
\text { T detector }=200^{\circ} \mathrm{C}\end{array}$ & [101] \\
\hline $\begin{array}{c}\text { 2-propenyl-ITC, } \\
\text { 3-(methylthio)propyl-ITC, } \\
\text { propyl ITC, 3-butenyl-ITC, } \\
\text { 3-butenenitrile, } \\
\text { 4-pentenenitrile, } \\
\text { 4-(methylsulfinyl)butyl- } \\
\text { ITC, } \\
\text { 5-(methylsulfinyl)- } \\
\text { pentanenitrile, } \\
\text { 3,4-epithiobutanenitrile }\end{array}$ & Broccoli seeds & $\begin{array}{l}\text { Seeds suspended in water } \\
\text { and ground in a mortar. } \\
\text { Washed with water and } \\
\text { incubated } \\
\text { at } 25^{\circ} \mathrm{C} \text { for } 2 \mathrm{~h} \text {. }\end{array}$ & $\mathrm{CH}_{2} \mathrm{Cl}_{2}$ & Centrifuge & GC-FID & $\begin{array}{l}\text { Column: HP5 } / 30 \mathrm{~m} \times \\
0.25 \mathrm{~mm} \times 0.25 \mu \mathrm{m}, \\
\text { splitless mode, } \\
\text { T injector }=200^{\circ} \mathrm{C}\end{array}$ & [102] \\
\hline $\begin{array}{c}\text { sec-butyl-ITC, } \\
\text { allyl-ITC, benzyl-ITC } \\
\text { 3-(methylthio)propyl-ITC, } \\
\text { 2-phenylethyl-ITC }\end{array}$ & Horseradish roots & $\begin{array}{l}\text { Samples were peeled } \\
\text { and ground. Addition of } \\
\mathrm{CH}_{2} \mathrm{Cl}_{2} \text { and vigorous } \\
\text { stirring for } 30 \mathrm{~min} .\end{array}$ & $\mathrm{CH}_{2} \mathrm{Cl}_{2}$ & $\begin{array}{c}\text { Filtration and } \\
\text { solvent-assisted flavor } \\
\text { evaporation at } 50^{\circ} \mathrm{C}\end{array}$ & HRGC-O/FID ${ }^{2}$ & $\begin{array}{c}\text { Column: DB-FFAP } / 30 \mathrm{~mm} \\
\times 0.32 \mathrm{~mm} \times 0.25 \mu \mathrm{m} \\
\text { or DB5 } / 30 \mathrm{~m} \times 0.32 \mathrm{~mm} \times \\
0.25 \mu \mathrm{m}, \\
\text { split ratio }(1: 1), \\
\text { T injection }=40^{\circ} \mathrm{C} \\
\text { (cold on-column } \\
\text { technique) }\end{array}$ & [103] \\
\hline $\begin{array}{l}\text { 4-(methylthio)butyl-ITC, } \\
\text { 2-phenylethyl-ITC, } \\
\text { 4-(methylthio)-3-butenyl- } \\
\text { ITC, } \\
\text { 4-methylpentyl-ITC, } \\
\text { benzyl-ITC, } \\
\text { 5-(methylthio)-4- } \\
\text { pentenenitrile, } \\
\text { benzenepropanenitrile }\end{array}$ & Radish & Clevenger hydrodistillation & $\mathrm{H}_{2} \mathrm{O}$ & - & GC-FID and GC-MS ${ }^{3}$ & $\begin{array}{c}\text { For GC-FID: } \\
\text { Column: HP-101/25 } \mathrm{m} \times \\
0.2 \mathrm{~mm} \times 0.2 \mu \mathrm{m} \\
\text { split ratio } 1: 50 \\
\text { T injector }=250^{\circ} \mathrm{C} \\
\text { T detector }=300^{\circ} \mathrm{C} \\
\text { For GC-MS: } \\
\text { Column: } \mathrm{HP}-20 \mathrm{M} / 50 \mathrm{~m} \times \\
0.2 \mathrm{~mm} \times 0.2 \mu \mathrm{m}, \\
\text { split ratio } 1: 50, \\
\mathrm{~T} \text { injector }=250{ }^{\circ} \mathrm{C}\end{array}$ & [108] \\
\hline
\end{tabular}


Table 4. Cont.

\begin{tabular}{|c|c|c|c|c|c|c|c|}
\hline Analyte & Vegetable & Sample Preparation & Extraction Solvent & Purification & Analytical Method & Conditions & Reference \\
\hline $\begin{array}{l}\text { 2-butyl-ITC, } \\
\text { phenylethyl-ITC }\end{array}$ & Indian mustard & $\begin{array}{l}\text { Dried and defatted seeds } \\
\text { and leaves mixed with } \\
\text { deionized water and } \\
\text { incubated at } 37^{\circ} \mathrm{C} \text { for } 2 \mathrm{~h} \text {. } \\
\text { Addition of myrosinase and } \\
\text { L-ascorbic acid. } \\
\text { Incubation for } 3 \mathrm{~h} \text { at } 37^{\circ} \mathrm{C} \text {. }\end{array}$ & $\mathrm{CH}_{2} \mathrm{Cl}_{2}$ & $\begin{array}{l}\text { Centrifuge for } 15 \mathrm{~min} \text { at } \\
4000 \mathrm{rpm} .\end{array}$ & GC-MS & $\begin{array}{c}\text { Column: } \mathrm{HP}-5 \mathrm{~m} / 0.25 \mathrm{~mm} \\
\times 30 \mathrm{~m} \times 0.25 \mu \mathrm{m}, \mathrm{split} \\
\text { ratio } 1: 50 \\
\mathrm{~T} \text { injector }=280^{\circ} \mathrm{C}\end{array}$ & [104] \\
\hline $\begin{array}{c}\text { sec-butyl-ITC, } \\
\text { 3-butenyl-ITC, } \\
\text { 4-pentenyl-ITC, } \\
\text { 4-(methylsulfanyl)butyl- } \\
\text { ITC, } \\
\text { 5-(methylsulfanyl)pentyl- } \\
\text { ITC, } \\
\text { 4-(methylsulfinyl)butyl- } \\
\text { ITC, } \\
\text { 2-phenylethyl-ITC, } \\
\text { 1-methoxyindole-3- } \\
\text { carbinol, } \\
\text { 3-methylpentanenitrile, } \\
\text { 4-pentenenitrile, } \\
\text { 3-hydroxypentenenitrile, } \\
\text { 5-hexenenitrile, } \\
\text { 5- } \\
\text { (methylsulfanyl)pentanenitrile, } \\
\text { 6- } \\
\text { (methylsulfanyl)hexanenitrile, } \\
\text { 5-(methylsulfinyl)- } \\
\text { pentanenitrile, } \\
\text { 3-phenylpropanenitrile, } \\
\text { indole-3-acetonitrile, } \\
\text { 1-methoxyindole-3- } \\
\text { acetonitrile, } \\
\text { 4,5-epithiopentanenitrile, } \\
\text { 3-hydroxy-4,5- } \\
\text { epithiopentanenitrile, } \\
\text { 5,6-epithiohexanenitrile, } \\
\text { 3-hydroxy-5,6- } \\
\text { epithiohexanenitrile, } \\
\text { 5-vinyl-1,3-oxazolidine-2- } \\
\text { thione }\end{array}$ & Brassica rapa leaves & $\begin{array}{l}\text { Fresh samples homogenized } \\
\text { with deionized water }\end{array}$ & $\mathrm{CH}_{2} \mathrm{Cl}_{2}$ & Centrifuge & GC-MS & $\begin{array}{c}\text { Column: SGE BP5MS } / 30 \mathrm{~m} \\
\times 0.25 \mathrm{~mm} \times 0.25 \mu \mathrm{m}, \\
\text { splitless mode, } \\
\mathrm{T} \text { injector }=190^{\circ} \mathrm{C}\end{array}$ & [105] \\
\hline
\end{tabular}


Table 4. Cont.

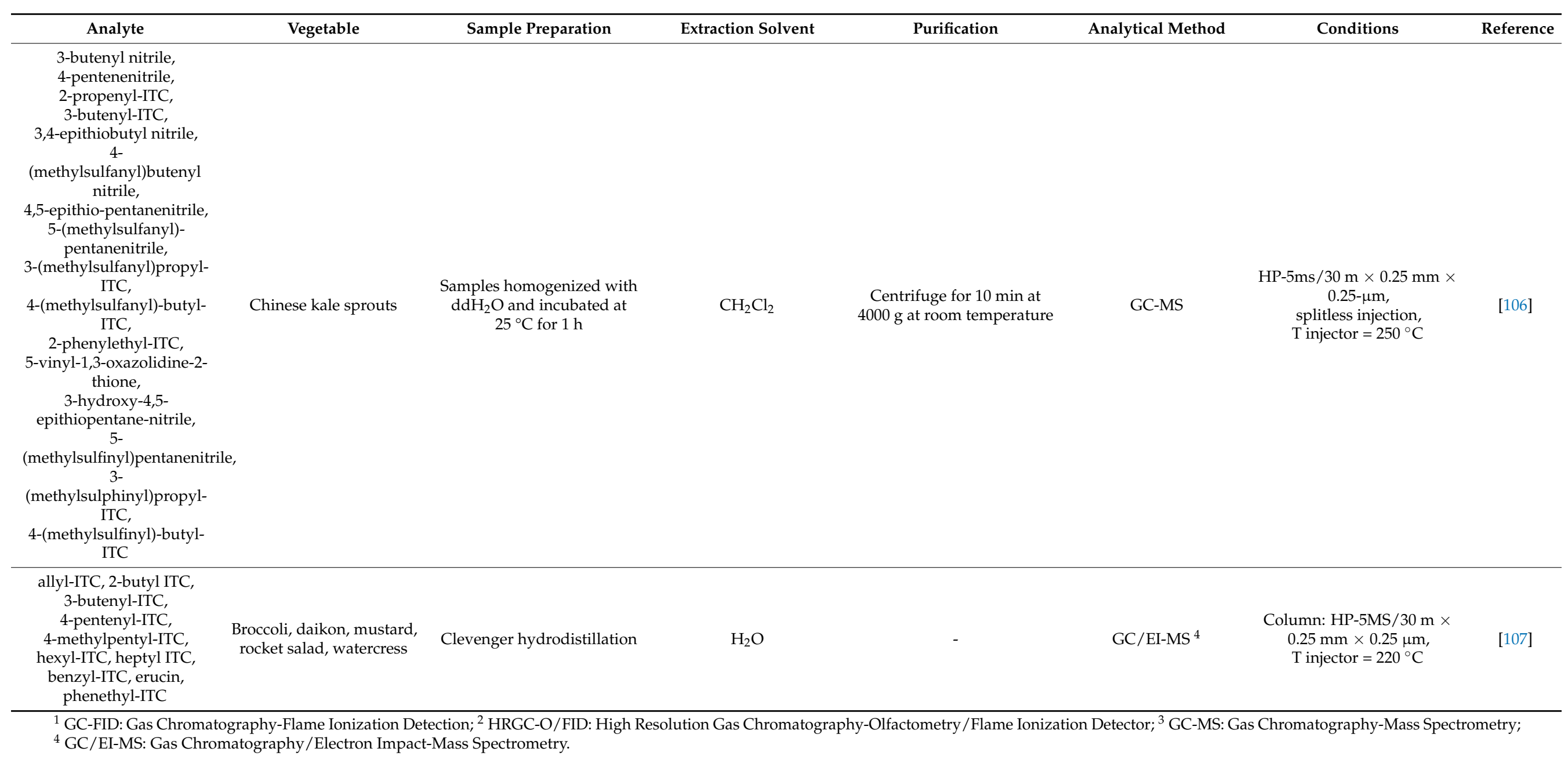


Glucosinolates can be subjected to enzymatic hydrolysis indirectly by an initial extraction of intact GLS. In this approach, the plant material is pulverized and the myrosinase enzyme is deactivated with liquid nitrogen or boiling water. Methanol or aqueous methanol is then employed to extract the intact GLS, which are desulfated on a diethylaminoethyl (DEAE) Sephadex column, with the addition of an artificial or natural myrosinase enzyme. This technique is advantageous primarily because of the possibility to adjust the column conditions, including $\mathrm{pH}$ (acidic, basic, or neutral) and temperature (low, room temperature, or high) depending on the type of hydrolysis product that is targeted to be obtained [59].

\subsection{Non-Conventional Extraction}

The conventional extraction methods are simple, especially for lab-scale processes; however, due to the large quantities of solvents and the long extraction times required, they may be replaced or enhanced by non-conventional extraction techniques such as High-Pressure Processing (HPP), High Voltage Electrical Discharges (HVED), UltrasoundAssisted Extraction (UAE), Microwave-Assisted Extraction (MAE), Supercritical Fluid Extraction (SFE), and Pressurized Fluid Extraction (PFE) (Table 5). Non-conventional extraction may be preferable, as it manages to reduce the extraction temperature and time, and therefore the consumption of the solvent, but at the same time it can achieve a higher efficiency and an improvement in the quality of the extracted compounds compared to the conventional methods. However, in the case of ITCs and indoles, the research for non-conventional extraction procedures is still ongoing and more studies are required for the establishment of these techniques as an alternative to conventional extraction.

The technique of HPP, (also known as High Hydrostatic Pressure (HHP)), is a nonthermal technology that utilizes liquids for a pressure transmission medium. Compared to thermal processing, HPP is more effective in the preservation of the flavor, texture, nutrients, and appearance of food products [109]. Applications of HPP in foods include the inactivation of microorganisms and enzymes and the increase in the shelf life of the products. Furthermore, the use of chemical preservatives is reduced [110]. After HPP on broccoli sprouts with $600 \mathrm{MPa}, 85 \%$ of GLS were converted to ITCs [111] which suggested the inactivation of the ESP protein. Moreover, the glucosinolate-myrosinase system in broccoli sprouts was not negatively affected. Two studies [110,112] have reported that the application of HHP at $400 \mathrm{MPa}$ in red cabbage and broccoli generated the highest amounts of ITCs. Moreover, it was found that the activity of myrosinase increased after processing, indicating that the increase in ITCs content is related to the stimulation of myrosinase activity [110].

Table 5. Non-conventional techniques applied for the extraction of isothiocyanates from cruciferous vegetables.

\begin{tabular}{|c|c|c|c|}
\hline Vegetable & Compounds Studied & Technology & References \\
\hline Red cabbage & Allyl-ITC & $\mathrm{HHP}^{1}$ & [112] \\
\hline Broccoli & Sulforaphane, erucin & HHP & [110] \\
\hline Broccoli sprouts & Iberin, sulforaphane, erucin & $\mathrm{HP}^{2}$ & [111] \\
\hline $\begin{array}{l}\text { Cauliflower } \\
\text { by-products }\end{array}$ & Isothiocyanates & $\mathrm{UAE}^{3}$ & [113] \\
\hline $\begin{array}{l}\text { Rapeseed, rapeseed } \\
\text { press-cake }\end{array}$ & Isothiocyanates & HVED $^{4}$ & [114] \\
\hline White cabbage & Sulforaphane & MAE $^{5}$ & [115] \\
\hline Wasabi & Allyl-ITC & SFE- $\mathrm{CO}_{2}{ }^{6}$ & [116] \\
\hline Horseradish & Allyl-ITC & SFE- $\mathrm{CO}_{2} /$ Hydrodistillation $/ \mathrm{H}_{2} \mathrm{O}$ & [117] \\
\hline Watercress & Isothiocyanates & $\mathrm{PFE}^{7}$ & [83] \\
\hline Garden cress & Benzyl-ITC & $\mathrm{UAE} / \mathrm{SFE}-\mathrm{CO}_{2} / \mathrm{ASE}^{8}$ & [118] \\
\hline
\end{tabular}

${ }^{1}$ HHP: High Hydrostatic Pressure ${ }^{2}$ HP: High Pressure ${ }^{3}$ UAE: Ultrasound-Assisted Extraction; ${ }^{4}$ HVED: High voltage electrical discharges;

${ }^{5}$ MAE: Microwave-Assisted Extraction; ${ }^{6}$ SFE- $\mathrm{CO}_{2}$ : Supercritical Fluid Extraction-Carbon Dioxide; ${ }^{7}$ PFE: Pressurized Fluid Extraction;

${ }^{8}$ ASE: Accelerated Solvent Extraction. 
High voltage electrical discharges are a non-thermal technique based on the electrical breakdown in water, while the air bubbles in water may accelerate the process. The HVED process initiates from the avalanche of electrons that is caused by the intense electrical field and constitutes the starting point of streamer propagation. The application of HVED improves the extraction yield of compounds through the destruction of the cellular structure and the enhancement of the mass transfer $[119,120]$. Barba et al. [114] employed HVED technology for the extraction of protein, polyphenols, and ITCs from rapeseeds and rapeseed press-cake. Different energy inputs $(0-400 \mathrm{~kJ} / \mathrm{kg})$ were tested, and the researchers concluded that the optimal treatment energy input was 80 or $240 \mathrm{~kJ} / \mathrm{kg}$, while when this was exceeded, the yield decreased. It is important to note that the nature of the sample influences the treatment efficiency to a great extent; thus, for each starting material, an optimization of the HVED process is required.

Ultrasound-assisted extraction (UAE) facilitates cell disruption and solvent penetration in the samples. This technique manages to enhance the extraction yield of compounds owing to the cavitation phenomena that are created by the ultrasound pressure waves in the solvent used for the extraction. Although the use of a solvent is required, the amount of solvent and energy consumption are reduced [121]. Besides, eco-friendly solvents such as natural deep eutectic solvents may be used with UAE to obtain high-quality extracts with increased yields [122]. Diatuo et al. [118] applied the UAE at a frequency of $24 \mathrm{kHz}$ in order to obtain extracts from cauliflower by-products that are rich in ITCs. The researchers developed a powder from the plant material, which was subjected to UAE. From the three solvents (distilled water, $70 \%$ methanol, and $80 \%$ acetonitrile) that were tested under UAE, the distilled water was the most efficient for the extraction of ITCs. The MAE is an alternative extraction procedure that has the potential to reduce the extraction time and the amount of solvent consumption while simultaneously increasing the extraction yield. Microwaves are electromagnetic waves with a frequency of $300 \mathrm{MHz}$ to $300 \mathrm{GHz}$. The process is based on the ionic conduction and dipole movement which are responsible for the warming of substances. The choice of the appropriate solvent in the MAE process is crucial. Solvents transparent to microwaves such as hexane do not heat up when subjected to the microwaves, while microwave absorbing solvents, such as ethanol or water, are more suitable for the MAE extraction process [123]. Tanongkankit et al. [115] studied the MAE of SFN from white cabbage in comparison to the conventional solvent extraction method. Different solvent types $\left(\mathrm{CH}_{2} \mathrm{Cl}_{2}\right.$ or water), microwave power, and extraction time were tested. MAE led to a higher extraction yield of SFN with a reduced extraction time compared to the classic solvent extraction method while both solvents, $\mathrm{CH}_{2} \mathrm{Cl}_{2}$, and water, provided similar yields.

In supercritical extraction, a supercritical fluid solvent, such as carbon dioxide, is used. The advantage of this technique is the avoidance of the use of organic solvents, which eventually reduces environmental concerns. The surface tension is not present in supercritical fluids and thus they have the potential to penetrate into small pores which are inaccessible to liquid solvents. This technique is very effective for the extraction of volatile compounds [124]. Li et al. [116] applied SFE for the extraction of allyl ITC from wasabi. The researchers tested different ranges of pressure $(15-25 \mathrm{MPa})$ and temperature $\left(35-55^{\circ} \mathrm{C}\right)$ and found that the extraction yield was increased with the increase in pressure and the decrease in temperature. The highest extraction yield was obtained at $25 \mathrm{MPa}$ and $35^{\circ} \mathrm{C}$, while the most significant parameters for the SFE process were pressure, temperature, and moisture content. Wu et al. [117] extracted allyl-ITC from horseradish using water extraction, hydrodistillation, and supercritical fluid extraction with carbon dioxide (SFE$\mathrm{CO}_{2}$ ). Hydrodistillation and SFE-CO $\mathrm{CO}_{2}$ provided similar extraction yields, whereas the conventional water extraction yield was significantly lower. Rafińska et al. [118] used UAE, SFE, and Accelerated Solvent Extraction (ASE) for the extraction of bioactive compounds from garden cress. It was found that the SFE method applied in dried garden cress sprouts was more efficient in the extraction of ITCs compared to ASE and UAE. The main ITC was found to be benzyl-ITC. 
Rodrigues et al. [83] reported the use of Pressurized Fluid Extraction (PFE) with supercritical $\mathrm{CO}_{2}$ for the recovery of ITCs and phenolic compounds from watercress. Pressurized fluid extraction is also an advantageous alternative method to the traditional extraction processes as it requires less solvent and a shorter extraction time. The results showed that the application of the PFE process was highly selective for the isolation of ITCs from watercress and the main isothiocyanate that was recovered was phenethyl-ITC. Moreover, an alternative extraction method for ITCs has been proposed using lactic acid bacteria (LAB). Jaiswal et al. [125] performed a fermentation-assisted extraction of ITCs from York cabbage using LAB. The researchers concluded that the employment of LAB facilitated the hydrolysis of GLS to the formation of ITCs. The factors with the highest influence in obtaining a high yield of ITCs were the fermentation time, the solid-to-liquid ratio, and the agitation rate. Yuan et al. [126] developed an ultrasound-assisted dispersivefilter extraction technique based on poly(deep eutectic solvent)-graphene oxide (PDES-GO) adsorbent for the isolation of I3C from broccoli. For the preparation of the PDES-GO, the researchers used a mixture of choline chloride and methacrylic acid in a 1:2 molar ratio in order to modify the graphene oxide surface. The specific method proved to be rapid, accurate, and low-cost. The efficiency of aqueous micellar systems (AMSs) was explored by Coscueta et al. [127] for the extraction of phenylethyl-ITC from watercress by-products. For this purpose, the surfactants Genapol X-080 (Clariant, Louisville, KY, USA) and Tergitol 15-S-7 (Sigma-Aldrich, Burlington, MA, USA) were utilized as it is known that they are effective in interacting with the low polar molecules of plant tissues. Liquid-liquid extraction was also performed using n-hexane and $\mathrm{MeCN} / \mathrm{CHCl}_{3}$. The highest content of phenylethyl-ITC was obtained using n-hexane; however, no statistically significant differences were observed between the extraction systems with Tergitol 15-S-7 (Sigma-Aldrich, Burlington, MA, USA) and $\mathrm{MeCN} / \mathrm{CHCl}_{3}$ compared to n-hexane, while the use of Genapol X-080 (Clariant, Louisville, KY, USA) afforded a slightly lower yield of phenylethyl-ITC.

\section{Methods of Analysis}

The analysis of ITCs and indoles has been performed using a great variety of chromatographic, spectrophotometric, and other techniques. However, the techniques that have been commonly employed are LC and GC, with or without the use of mass spectrometry detection (Tables 3 and 4). Furthermore, prior to the analysis of ITCs, derivatization may be applied owing to the high volatility and instability of these compounds and the absence of chromophore groups.

\subsection{Spectroscopic Techniques}

\subsubsection{UV-Vis Spectrophotometry}

Spectrophotometry is defined as the measurement of the absorption or reflectance properties of a substance as a function of the wavelength. The key advantages of the UV-Vis spectrophotometry methods are the low analysis time and the reduced labor consumption, while they simultaneously exhibit a high sensitivity and precision [128]. A UV-Vis spectrophotometric method for the determination of total ITCs content has been developed [44,129]. This method is indirect, and it is based on the quantitative reaction of ITCs with an excess of 1,2-benzenedithiol (Figure 4). The product of the cyclocondensation reaction, 1,3-benzodithiole-2-thione, is determined spectrophotometrically at $365 \mathrm{~nm}$.

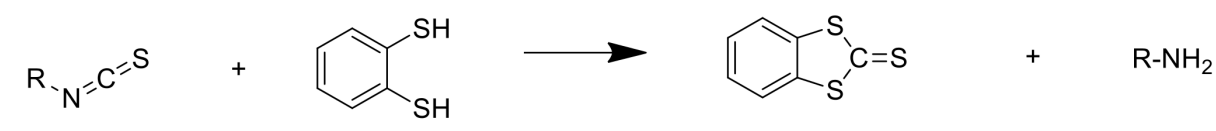

Figure 4. Cyclocondensation reaction of isothiocyanates with 1,2-benzenedithiol. 
The reaction of 1,2-benzenedithiol with ITCs is highly selective; thus, other enzymatic hydrolysis products of GLS, such as thiocyanates, do not react. Although the sensitivity of the method is very low $(1 \mathrm{nmol})$, it can be considered a time-consuming method of analysis due to the pre-required stage of derivatization. However, this cyclocondensation reaction has been utilized in several studies for the determination of total ITCs with High-Performance Liquid Chromatography (HPLC) [21,130,131] or GC-MS [132].

\subsubsection{Fourier-Transform Infrared Spectroscopy (FT-IR)}

Another method that has been reported in the literature for the determination of the total ITCs in cruciferous vegetables is the Attenuated Total Reflectance Fourier-Transform Infrared Spectroscopy (ATR-FTIR) method in combination with the partial least-squares (PLS) algorithm [45]. The sampling technique of ATR is advantageous as it enables the direct examination of samples without the requirement of a derivatization step [133]. The spectral range $2150-2020 \mathrm{~cm}^{-1}$ was used for the quantification of total ITCs in broccoli and the results showed that it is an equivalent method in terms of reproducibility and accuracy to the UV-Vis spectrophotometric method [44]. This method seems to be simple and rapid and constitutes a useful alternative for the determination of total ITCs in cruciferous vegetables.

\subsection{Chromatographic Techniques}

\subsubsection{Paper Chromatography (PC)}

Paper chromatography is the oldest method used to analyze GLS hydrolysis products $[134,135]$. Ammonia reacts with ITCs and produces thiourea-type derivatives which are separated by PC. The components that are hydrophobic are analyzed using water saturated $\mathrm{CHCl}_{3}$, while the hydrophilic substances are analyzed with a mixture of water, butanol, and toluene as the developing solvent system. However, PC exhibits low reproducibility for the analysis of ITCs; thus, alternative chromatographic methods have been developed for their analysis [136].

\subsubsection{Thin Layer Chromatography (TLC)}

Thin layer chromatography has been used to determine the compounds 1-isothiocyanato-4-(methylsulfinyl)butane, 1-isothiocyanato-3-(methylsulfinyl)propane, 5-(methylsulfinyl)pentanenitrile, and 4-(methylsulfinyl)butanenitrile, from broccoli and Lesquerella fendleri [137].

\subsubsection{Gas Chromatography (GC)}

Gas chromatography is an excellent separation technique for the identification and determination of volatile organic substances. This technique in combination with a Flame Ionization Detector (FID) has been used for the determination of SFN [29,138-140] and other ITCs [100-103,108] in a great variety of cruciferous vegetables. However, the thermal degradation of SFN to 3-butenyl ITC has been observed under split/splitless conditions [31]. Besides, the thermal degradation studies that have been performed for ITCs show that these compounds are thermolabile [32,141,142]. Therefore, LC methods are often preferred for their analysis.

\subsubsection{High-Performance Liquid Chromatography (HPLC)}

For the analysis of ITCs and certain indoles, HPLC coupled with a UV detector has been proposed by various researchers $[19,25,78,88,92,93,95-97,99]$. For the determination of I3C, indole-3-acetonitrile, and 3,3'-diindolylmethane, HPLC with a Fluorescence Detector (FLD) is usually employed $[87,99,143]$. The determination is performed at excitation at 280-285 nm, and emission at $340 \mathrm{~nm}$. In the case of SFN, the UV detection has been performed at $202 \mathrm{~nm}$ [95], $205 \mathrm{~nm}$ [93], and $196 \mathrm{~nm}$ [19]. However, the lack of strong UV chromophores in several ITCs, and especially SFN, creates analytical problems. For this reason, Nakagawa et al. [144] and Liang et al. [20] proposed the use of an Evaporative Light- 
Scattering Detector (ELSD) for analyzing SFN, achieving a higher sensitivity compared to a UV detector. Another problem that also arises in the HPLC analysis of ITCs is their precipitation when aqueous mobile phases are used. This impacts the accuracy of determinations and leads to operational problems [22].

\subsubsection{High Speed Counter Current Chromatography (HSCCC)}

High speed counter current chromatography is a simple and low-cost method which is based on the distribution of substances between two immiscible liquids. This technique enables the recovery and purification of samples; thus, it can be used for the isolation of natural products from plant extracts $[145,146]$. Liang et al., applied HSCCC in broccoli seed meal in order to purify and recover SFN [147]. The two-phase liquid system used in this study was n-hexane/ethyl acetate/methanol/water (1:5:1:5, $v / v / v / v)$ which was selected based on the partition coefficient of SFN. The results of HSCCC were compared with the preparative HPLC method. Sulforaphane was obtained with a slightly higher purity (97\%) via HSCCC compared to preparative HPLC (95\%), while the recovery obtained from HSCCC was higher $(98.5 \%)$ than the preparative HPLC method $(87.4 \%)$. High speed counter current chromatography has been also employed for the purification of sulforaphene from radish seeds [148]. The two-phase solvent system used in this study was n-hexane-ethyl acetate-methanol-water (35:100:35:100, v/v/v/v). High purity sulforaphene was obtained $(96.9 \%)$, while the recovery of the compound was $95.2 \%$.

\subsubsection{Supercritical Fluid Chromatography (SFC)}

Supercritical fluid chromatography constitutes a variation of HPLC. The difference between HPLC and SFC lies in the fact that in SFC, a supercritical fluid is used instead of the liquid mobile phase. This technique is considered a good alternative to HPLC, as the properties of the supercritical fluid used in the mobile phase is between a liquid and a gas. Supercritical $\mathrm{CO}_{2}$ is commonly employed owing to its low cost, the fact that it is safe to use in the food industry, and also because it has no negative impact on the environment [149]. Supercritical fluid chromatography has been used for the analysis of the hydrolysis products of indole GLS. Buskov et al. [150] used SFC for the determination of ascorbigens in the autolysates of broccoli, white cabbage, red cabbage, cauliflower, Brussels sprouts, and various Portuguese cabbages, while Buskov et al. [151] studied the degradation products of 4-hydroxybenzylglucosinolate. The study of the hydrolysis products of indol3-ylmethyl-glucosinolates in broccoli heads by SFC has been also reported [152].

\subsection{Hyphenated and Other Techniques}

\subsubsection{Liquid Chromatography /Mass Spectrometry}

The combination of mass spectrometry with liquid chromatography has been successfully applied for the determination of ITCs and indoles. Baenas et al. [89] used the Ultrahigh Performance Liquid Chromatography method coupled with Triple Quadrupole Mass Spectrometry/Mass Spectrometry (UHPLC-QqQ-MS/MS) for the determination of SFN and iberin in cooked broccolini and kale. Andini et al. [94] and Pilipczuk [153] analyzed ITCs with Ultra-High-Performance Liquid Chromatography-Mass Spectrometry (UHPLC-MS), applying a derivatization method using N-acetyl-L-cysteine (NAC) (Figure 5), achieving very low detection limits (0.9-2.6 $\mu \mathrm{M})$ [94].

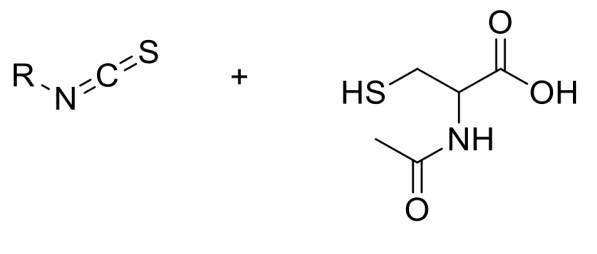

Figure 5. Derivatization reaction of isothiocyanates with $\mathrm{N}$-acetyl-L-cysteine. 
High resolution mass spectrometry has been used for the qualitative and quantitative determination of ITCs and indoles. A mass spectrometry study of SFN and indole-3-carbinol has been performed by Kokotou et al. [42] using Ultraperformance Liquid Chromatography-High Resolution Mass Spectrometry/Mass Spectrometry (UPLCHRMS/MS) avoiding a derivatization step. The researchers also developed a method for the simultaneous determination of compounds in broccoli extracts.

The simultaneous determination of I3C, indole-3-carboxaldehyde, ascorbigen, and indole-3-acetic acid by Liquid Chromatography-Quadrupole-Time of Flight-Mass Spectrometry (LC-Q-TOF-MS) in a broad range of cruciferous vegetables has been also reported [43].

\subsubsection{Gas Chromatography/Mass Spectrometry}

The determination of ITCs with GC-MS has been employed [104-107,154-156]. For the analysis of volatile ITCs, the eco-friendly technique of Solid Phase Microextraction (SPME) in conjunction with GC-MS has been also proposed [157-161]. A method employing GC-MS with a pre-column derivatization step has been also developed by Latxague et al. [26].

\subsubsection{Other Techniques}

Gonda et al., reported the development of a capillary electrophoresis (CE) method for the simultaneous quantification of sinigrin, gluconasturtiin, and allyl-ITC [162]. The ITCs are determined after the myrosinase hydrolysis of GLS and derivatization of ITCs in-vial, utilizing mercaptoacetic acid (Figure 6). The analysis was performed in extracts of cruciferous vegetables such as Brussels sprouts, radish, horseradish, and watercress. This method has the advantage that it can be used to determine both GLS and ITCs, and it also requires a minimum sample size.

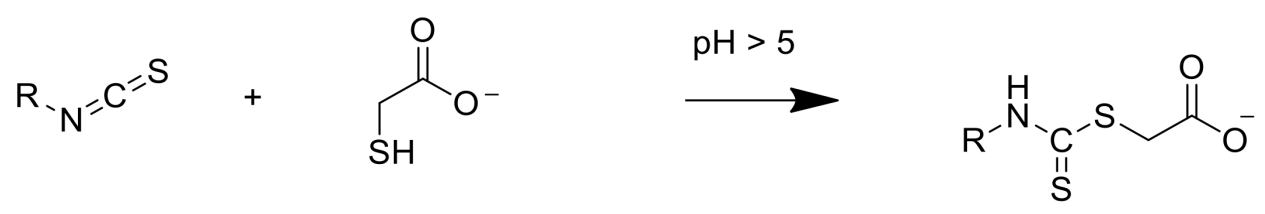

\section{Isothiocyanate Mercaptoacetic acid}

Figure 6. Derivatization of isothiocyanates with mercaptoacetic acid.

\section{Conclusions}

Since ITCs and indoles are products of enzymatic hydrolysis, the sample preparation procedure is considered crucial for their determination. The hydrolysis of glucosinolates by myrosinase should be complete and the conditions must be in favor of producing ITCs and indoles, rather than other compounds with an increased toxicity. Following the sample preparation, the extraction solvents that are commonly used are chlorinated. The toxicity of the extraction solvents poses limitations in the use of extracts in the development of food products enriched with these health-promoting compounds. Considering the solvent toxicity, future research should be focused on the employment of eco-friendly solvents, such as natural deep eutectic solvents, which are still unexplored in this field. These efforts may be enhanced by the use of non-conventional techniques, such as ultrasound and microwaveassisted extraction. Further research on the development of non-conventional techniques is required towards the control and optimization of the processing conditions, as the treatment efficiency may be affected by the nature of the raw materials. The analytical determination of ITCs and indoles is commonly performed by liquid or gas chromatography. Nevertheless, the determination of ITCs presents analytical problems such as the precipitation in the liquid chromatography column and the weak DAD signal, while in gas chromatography certain ITCs can be degraded. These problems are attributed to the high volatility, instability, and the lack of chromophores which can be addressed by the use of derivatization reagents 
and the employment of hyphenated mass spectrometry methods. Due to the differences in the chemical properties between ITCs and indoles, the analytical methods for the simultaneous determination of these compounds in cruciferous vegetables are scarce, and therefore additional analytical methods must be developed for this purpose.

Author Contributions: Conceptualization, P.-K.R. and S.K.; writing—original draft preparation S.K. and P.-K.R.; writing - review and editing, S.K., P.-K.R., M.X., M.G.K. and V.C.-K.; Critical revising, M.X., M.G.K. and V.C.-K. All authors have read and agreed to the published version of the manuscript.

Funding: This research received no external funding.

Institutional Review Board Statement: Not applicable.

Data Availability Statement: Not applicable.

Conflicts of Interest: The authors declare no conflict of interest.

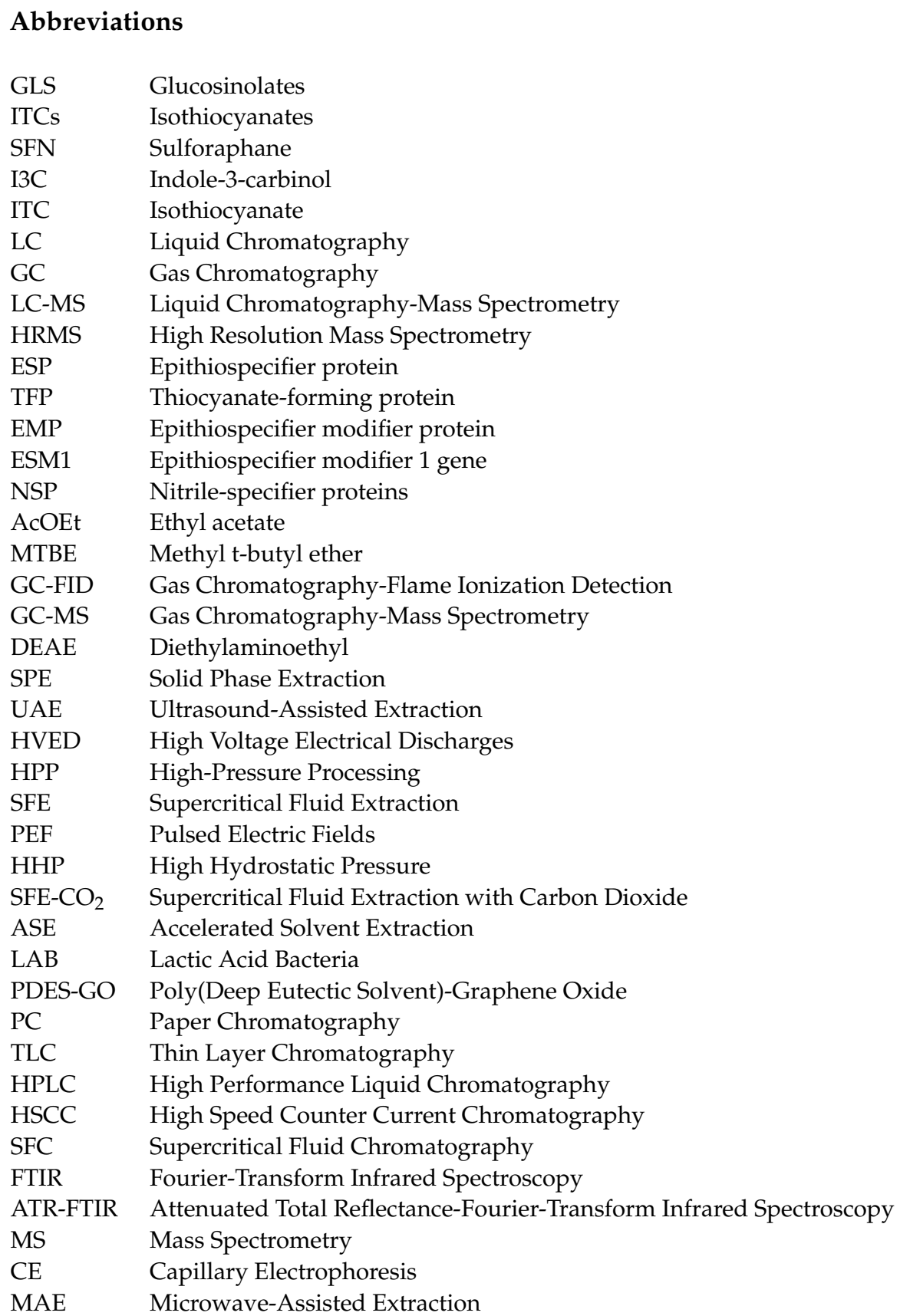




$\begin{array}{ll}\text { HRGC-O/FID } & \text { High Resolution Gas Chromatography-Olfactometry/Flame Ionization } \\ & \text { Detector } \\ \text { GC/EI-MS } & \text { Gas Chromatography/Electron Impact-Mass Spectrometry } \\ \text { UHPLC-QqQ-MS/MS } & \begin{array}{l}\text { Ultrahigh Performance Liquid Chromatography-Triple Quadruple-Mass } \\ \text { Spectrometry/Mass Spectrometry }\end{array} \\ \text { UHPLC-MS } & \text { Ultra-High-Performance Liquid Chromatography-Mass Spectrometry } \\ \text { NAC } & \text { N-acetyl-L-cysteine } \\ \text { UPLC-HRMS/MS } & \text { Ultraperformance Liquid Chromatography-High Resolution Mass } \\ & \text { Spectrometry/Mass Spectrometry } \\ \text { SPME } & \text { Solid Phase Microextraction } \\ \text { PLS } & \text { Partial Least-Squares } \\ \text { FLD } & \text { Fluorescence Detector } \\ \text { ELSD } & \text { Evaporative Light-Scattering Detector } \\ \text { HPLC-UV } & \text { High Performance Liquid Chromatography-Ultraviolet Detector } \\ \text { HPLC-DAD-FLD } & \text { High Performance Liquid Chromatography-Diode-Array Detection- } \\ & \text { Fluorescence Detector } \\ \text { HPLC-DAD } & \text { High Performance Liquid Chromatography-Diode-Array Detection } \\ \text { HPLC-PDA-UV } & \text { High Performance Liquid Chromatography-Photodiode-Array Detection- } \\ & \text { Ultraviolet Detector } \\ \text { RP-UHPLC-ESI-MS } & \text { Reverse Phase-Ultrahigh Performance Liquid Chromatography-Electronspray } \\ & \text { Ionization-Mass Spectrometry } \\ \text { LC-Q-TOF-MS } & \text { Liquid Chromatography-Quadruple-Time of Flight-Mass Spectrometry }\end{array}$

\section{References}

1. Favela-González, K.M.; Hernández-Almanza, A.Y.; De la Fuente-Salcido, N.M. The value of bioactive compounds of cruciferous vegetables (Brassica) as antimicrobials and antioxidants: A review. J. Food Biochem. 2020, 44, e13414. [CrossRef] [PubMed]

2. Zhang, Y. Cancer-preventive isothiocyanates: Measurement of human exposure and mechanism of action. Mutat. Res. Mol. Mech. Mutagen. 2004, 555, 173-190. [CrossRef] [PubMed]

3. Vazquez-Prieto, M.A.; Miatello, R.M. Organosulfur compounds and cardiovascular disease. Mol. Asp. Med. 2010, 31, 540-545. [CrossRef] [PubMed]

4. Bachiega, P.; Salgado, J.M.; de Carvalho, J.E.; Ruiz, A.L.T.G.; Schwarz, K.; Tezotto, T.; Caldeira-Morzelle, M. Antioxidant and antiproliferative activities in different maturation stages of broccoli (Brassica oleracea Italica) biofortified with selenium. Food Chem. 2016, 190, 771-776. [CrossRef]

5. Mazarakis, N.; Snibson, K.; Licciardi, P.V.; Karagiannis, T.C. The potential use of 1-sulforaphane for the treatment of chronic inflammatory diseases: A review of the clinical evidence. Clin. Nutr. 2020, 39, 664-675. [CrossRef]

6. Briones-Herrera, A.; Eugenio-Pérez, D.; Reyes-Ocampo, J.G.; Rivera-Mancía, S.; Pedraza-Chaverri, J. New highlights on the health-improving effects of sulforaphane. Food Funct. 2018, 9, 2589-2606. [CrossRef]

7. Gründemann, C.; Huber, R. Chemoprevention with isothiocyanates-from bench to bedside. Cancer Lett. 2018, 414, 26-33. [CrossRef]

8. Ramirez, D.; Abellán-Victorio, A.; Beretta, V.; Camargo, A.; Moreno, D.A. Functional ingredients from Brassicaceae species: Overview and perspectives. Int. J. Mol. Sci. 2020, 21, 1998. [CrossRef]

9. Chhajed, S.; Mostafa, I.; He, Y.; Abou-Hashem, M.; El-Domiaty, M.; Chen, S. Glucosinolate biosynthesis and the glucosinolatemyrosinase system in plant defense. Agronomy 2020, 10, 1786. [CrossRef]

10. Agrawal, A.A.; Kurashige, N.S. A role for isothiocyanates in plant resistance against the specialist herbivore Pieris rapae. J. Chem. Ecol. 2003, 29, 1403-1415. [CrossRef]

11. Plaszkó, T.; Szúcs, Z.; Vasas, G.; Gonda, S. Effects of glucosinolate-derived isothiocyanates on fungi: A comprehensive review on direct effects, mechanisms, structure-activity relationship data and possible agricultural applications. J. Fungi 2021, 7, 539. [CrossRef] [PubMed]

12. Liang, H.; Yuan, Q. Natural sulforaphane as a functional chemopreventive agent: Including a review of isolation, purification and analysis methods. Crit. Rev. Biotechnol. 2012, 32, 218-234. [CrossRef]

13. Pinz, S.; Unser, S.; Rascle, A. The natural chemopreventive agent sulforaphane inhibits STAT5 activity. PLoS ONE 2014,9 , e99391. [CrossRef] [PubMed]

14. McDanell, R.; McLean, A.; Hanley, A.; Heaney, R.; Fenwick, G. Chemical and biological properties of indole glucosinolates (glucobrassicins): A review. Food Chem. Toxicol. 1988, 26, 59-70. [CrossRef]

15. Ruiz, R.B.; Hernández, P.S. Cancer chemoprevention by dietary phytochemicals: Epidemiological evidence. Maturitas 2016, 94, 13-19. [CrossRef]

16. Chinni, S.R.; Li, Y.; Upadhyay, S.; Koppolu, P.K.; Sarkar, F.H. Indole-3-carbinol (I3C) induced cell growth inhibition, G1 cell cycle arrest and apoptosis in prostate cancer cells. Oncogene 2001, 20, 2927-2936. [CrossRef] 
17. Megna, B.W.; Carney, P.R.; Nukaya, M.; Geiger, P.; Kennedy, G.D. Indole-3-carbinol induces tumor cell death: Function follows form. J. Surg. Res. 2016, 204, 47-54. [CrossRef] [PubMed]

18. Pelosi, C.; Chiron, F.; Dubs, F.; Hedde, M.; Ponge, J.-F.; Salmon, S.; Cluzeau, D.; Nélieu, S. A new method to measure allyl isothiocyanate (AITC) concentrations in mustard-Comparison of AITC and commercial mustard solutions as earthworm extractants. Appl. Soil Ecol. 2014, 80, 1-5. [CrossRef]

19. Ares, A.M.; Bernal, J.; Martín, M.T.; Nozal, M.J. Optimized formation, extraction, and determination of sulforaphane in broccoli by liquid chromatography with diode array detection. Food Anal. Methods 2014, 7, 730-740. [CrossRef]

20. Liang, H.; Yuan, Q.; Liu, M. Simultaneous determination of glucoraphanin and sulforaphane in Brassica oleracea seeds by high-performance liquid chromatography with evaporative light-scattering detector. Nat. Prod. Res. 2013, 27, 194-197. [CrossRef]

21. Fusari, C.M.; Locatelli, D.A.; Altamirano, J.; Camargo, A.B. UAE-HPLC-UV: New contribution for fast determination of total isothiocyanates in Brassicaceae vegetables. J. Chem. 2015, 2015, e294601. [CrossRef]

22. Wilson, E.A.; Ennahar, S.; Marchioni, E.; Bergaentzlé, M.; Bindler, F. Improvement in determination of isothiocyanates using high-temperature reversed-phase HPLC. J. Sep. Sci. 2012, 35, 2026-2031. [CrossRef] [PubMed]

23. Ciska, E.; Drabińska, N.; Narwojsz, A.; Honke, J. Stability of glucosinolates and glucosinolate degradation products during storage of boiled white cabbage. Food Chem. 2016, 203, 340-347. [CrossRef] [PubMed]

24. Masumoto, N.; Nishizaki, Y.; Nakajima, K.; Sugimoto, N.; Sato, K. Determination of allyl isothiocyanate in mustard and horseradish extracts by single reference GC and HPLC based on relative molar sensitivities. J. Food Hyg. Soc. Jpn. 2021, 62, 73-78, [CrossRef]

25. Tang, L.; Paonessa, J.D.; Zhang, Y.; Ambrosone, C.B.; McCann, S.E. Total isothiocyanate yield from raw cruciferous vegetables commonly consumed in the United States. J. Funct. Foods 2013, 5, 1996-2001. [CrossRef]

26. Latxague, L.; Gardrat, C.; Coustille, J.; Viaud, M.; Rollin, P. Identification of enzymatic degradation products from synthesized glucobrassicin by gas chromatography-mass spectrometry. J. Chromatogr. A 1991, 586, 166-170. [CrossRef]

27. Tolonen, M.; Taipale, M.; Viander, B.; Pihlava, J.-M.; Korhonen, H.; Ryhänen, E.-L. Plant-derived biomolecules in fermented cabbage. J. Agric. Food Chem. 2002, 50, 6798-6803. [CrossRef]

28. Farag, M.A.; Motaal, A.A.A. Sulforaphane composition, cytotoxic and antioxidant activity of crucifer vegetables. J. Adv. Res. 2010, 1, 65-70. [CrossRef]

29. Moon, J.-K.; Kim, J.-R.; Ahn, Y.-J.; Shibamoto, T. Analysis and anti-helicobacter activity of sulforaphane and related compounds present in broccoli (Brassica oleracea L.) sprouts. J. Agric. Food Chem. 2010, 58, 6672-6677. [CrossRef]

30. Klopsch, R.; Witzel, K.; Börner, A.; Schreiner, M.; Hanschen, F. Metabolic profiling of glucosinolates and their hydrolysis products in a germplasm collection of Brassica rapa turnips. Food Res. Int. 2017, 100, 392-403. [CrossRef]

31. Chiang, W.C.K.; Pusateri, A.D.J.; Leitz, R.E.A. Gas chromatography/mass spectrometry method for the determination of sulforaphane and sulforaphane nitrile in broccoli. J. Agric. Food Chem. 1998, 46, 1018-1021. [CrossRef]

32. Chen, C.-W.; Rosen, R.T.; Ho, C.-T. Analysis of thermal degradation products of allyl isothiocyanate and phenethyl isothiocyanate. In Flavor Analysis; ACS Symposium Series; American Chemical Society: Washington, DC, USA, 1998; Volume 705, pp. 152-166, ISBN 978-0-8412-3578-6.

33. Song, L.; Iori, R.; Thornalley, P.J. Purification of major glucosinolates from Brassicaceae seeds and preparation of isothiocyanate and amine metabolites. J. Sci. Food Agric. 2006, 86, 1271-1280. [CrossRef]

34. Franco, P.; Spinozzi, S.; Pagnotta, E.; Lazzeri, L.; Ugolini, L.; Camborata, C.; Roda, A. Development of a liquid chromatographyelectrospray ionization-tandem mass spectrometry method for the simultaneous analysis of intact glucosinolates and isothiocyanates in Brassicaceae seeds and functional foods. J. Chromatogr. A 2016, 1428, 154-161. [CrossRef] [PubMed]

35. Oliviero, T.; Verkerk, R.; Vermeulen, M.; Dekker, M. In Vivo formation and bioavailability of isothiocyanates from glucosinolates in broccoli as affected by processing conditions. Mol. Nutr. Food Res. 2014, 58, 1447-1456. [CrossRef] [PubMed]

36. Ares, A.M.; Valverde, S.; Bernal, J.L.; Nozal, M.J. Development and validation of a LC-MS/MS method to determine sulforaphane in honey. Food Chem. 2015, 181, 263-269. [CrossRef]

37. Al Janobi, A.A.; Mithen, R.; Gasper, A.V.; Shaw, N.; Middleton, R.J.; Ortori, C.A.; Barrett, D.A. Quantitative measurement of sulforaphane, iberin and their mercapturic acid pathway metabolites in human plasma and urine using liquid chromatographytandem electrospray ionisation mass spectrometry. J. Chromatogr. B 2006, 844, 223-234. [CrossRef]

38. Dominguez-Perles, R.; Medina, S.; Moreno-Fernández, D.Á.; Garcia-Viguera, C.; Ferreres, F.; Gil-Izquierdo, Á. A new ultra-rapid UHPLC/MS/MS method for assessing glucoraphanin and sulforaphane bioavailability in human urine. Food Chem. 2014, 143, 132-138. [CrossRef] [PubMed]

39. Hauder, J.; Winkler, S.; Bub, A.; Rüfer, C.E.; Pignitter, M.; Somoza, V. LC-MS/MS quantification of sulforaphane and indole-3carbinol metabolites in human plasma and urine after dietary intake of selenium-fortified broccoli. J. Agric. Food Chem. 2011, 59, 8047-8057. [CrossRef]

40. Zheng, L.; Zheng, F. Development and validation of an LC-APCI-MS/MS method for the determination of phenethyl isothiocyanate in human plasma. Biomed. Chromatogr. 2014, 29, 619-625. [CrossRef]

41. Platz, S.; Piberger, A.L.; Budnowski, J.; Herz, C.; Schreiner, M.; Blaut, M.; Hartwig, A.; Lamy, E.; Hanske, L.; Rohn, S. Bioavailability and biotransformation of sulforaphane and erucin metabolites in different biological matrices determined by LC-MS-MS. Anal. Bioanal. Chem. 2015, 407, 1819-1829. [CrossRef] 
42. Kokotou, M.G.; Revelou, P.-K.; Pappas, C.; Constantinou-Kokotou, V. High resolution mass spectrometry studies of sulforaphane and indole-3-carbinol in broccoli. Food Chem. 2017, 237, 566-573. [CrossRef]

43. Revelou, P.-K.; Kokotou, M.G.; Constantinou-Kokotou, V. Determination of indole-type phytonutrients in cruciferous vegetables. Nat. Prod. Res. 2018, 34, 2554-2557. [CrossRef]

44. Zhang, Y.; Cho, C.-G.; Posner, G.H.; Talalay, P. Spectroscopic quantitation of organic isothiocyanates by cyclocondensation with vicinal dithiols. Anal. Biochem. 1992, 205, 100-107. [CrossRef]

45. Revelou, P.; Kokotou, M.; Pappas, C.; Constantinou-Kokotou, V. Direct determination of total isothiocyanate content in broccoli using attenuated total reflectance infrared Fourier transform spectroscopy. J. Food Compos. Anal. 2017, 61, 47-51. [CrossRef]

46. Thornalley, P. Cruciferous Vegetables, Isothiocyanates and Indoles; IARC: Lyon, France, 2004; pp. 1-250.

47. Šamec, D.; Salopek-Sondi, B. Chapter 3.11-Cruciferous (Brassicaceae) vegetables. In Nonvitamin and Nonmineral Nutritional Supplements; Nabavi, S.M., Silva, A.S., Eds.; Academic Press: Cambridge, UK, 2019; pp. 195-202, ISBN 978-0-12-812491-8.

48. Liu, B.; Mao, Q.; Cao, M.; Xie, L. Cruciferous vegetables intake and risk of prostate cancer: A meta-analysis. Int. J. Urol. 2011, 19, 134-141. [CrossRef]

49. Liu, X.; Lv, K. Cruciferous vegetables intake is inversely associated with risk of breast cancer: A meta-analysis. Breast 2013, 22, 309-313. [CrossRef] [PubMed]

50. Liu, B.; Mao, Q.; Lin, Y.; Zhou, F.; Xie, L. The association of cruciferous vegetables intake and risk of bladder cancer: A meta-analysis. World J. Urol. 2012, 31, 127-133. [CrossRef] [PubMed]

51. Wu, Q.-J.; Yang, Y.; Wang, J.; Han, L.-H.; Xiang, Y.-B. Cruciferous vegetable consumption and gastric cancer risk: A meta-analysis of epidemiological studies. Cancer Sci. 2013, 104, 1067-1073. [CrossRef] [PubMed]

52. Liu, B.; Mao, Q.; Wang, X.; Zhou, F.; Luo, J.; Wang, C.; Lin, Y.; Zheng, X.; Xie, L. Cruciferous vegetables consumption and risk of renal cell carcinoma: A meta-analysis. Nutr. Cancer 2013, 65, 668-676. [CrossRef]

53. Murillo, G.; Mehta, R.G. Cruciferous vegetables and cancer prevention. Nutr. Cancer 2001, 41, 17-28. [CrossRef]

54. Jahangir, M.; Kim, H.K.; Choi, Y.H.; Verpoorte, R. Health-affecting compounds in Brassicaceae. Compr. Rev. Food Sci. Food Saf. 2009, 8, 31-43. [CrossRef]

55. Šamec, D.; Pavlović, I.; Salopek-Sondi, B. White cabbage (Brassica oleracea var. capitata f. alba): Botanical, phytochemical and pharmacological overview. Phytochem. Rev. 2017, 16, 117-135. [CrossRef]

56. Han, B.; Li, X.; Yu, T. Cruciferous vegetables consumption and the risk of ovarian cancer: A meta-analysis of observational studies. Diagn. Pathol. 2014, 9, 7. [CrossRef] [PubMed]

57. Latté, K.P.; Appel, K.-E.; Lampen, A. Health benefits and possible risks of broccoli-an overview. Food Chem. Toxicol. 2011, 49, 3287-3309. [CrossRef]

58. Vig, A.P.; Rampal, G.; Thind, T.S.; Arora, S. Bio-protective effects of glucosinolates—a review. LWT 2009, 42, 1561-1572. [CrossRef]

59. Mérillon, J.-M.; Ramawat, K.G. Reference Series in Phytochemistry; Mérillon, J.-M., Ramawat, K.G., Eds.; Springer: Berlin/Heidelberg, Germany, 2017; ISBN 978-3-319-25461-6.

60. Kushad, M.M.; Brown, A.F.; Kurilich, A.C.; Juvik, J.A.; Klein, B.P.; Wallig, M.A.; Jeffery, E. Variation of glucosinolates in vegetable crops of Brassica oleracea. J. Agric. Food Chem. 1999, 47, 1541-1548. [CrossRef]

61. Pedras, M.S.C.; Yaya, E.E. Phytoalexins from Brassicaceae: News from the front. Phytochemistry 2010, 71, 1191-1197. [CrossRef]

62. Slovin, J.P.; Bandurski, R.S.; Cohen, J.D. Chapter 5-Auxin. In New Comprehensive Biochemistry; Hooykaas, P.J.J., Hall, M.A., Libbenga, K.R., Eds.; Elsevier: Amsterdam, The Netherlands, 1999; Volume 33, pp. 115-140, ISBN 0167-7306.

63. Kala, C.; Khan, N.A. Isolation and characterization of isopropyl isothiocyanate isolated from seeds of Drypetes roxburghii wall and its anti-platelet and anti-thrombotic activity. Sci. Afr. 2020, 10, e00658. [CrossRef]

64. Johnson, S.D.; Griffiths, M.E.; Peter, C.I.; Lawes, M.J. Pollinators, "mustard oil" volatiles, and fruit production in flowers of the dioecious tree Drypetes natalensis (Putranjivaceae). Am. J. Bot. 2009, 96, 2080-2086. [CrossRef]

65. Møller, B.L. Functional diversifications of cyanogenic glucosides. Curr. Opin. Plant Biol. 2010, 13, 337-346. [CrossRef]

66. Bennett, R.N.; Kiddle, G.; Wallsgrove, R.M. Biosynthesis of benzylglucosinolate, cyanogenic glucosides and phenylpropanoids in Carica papaya. Phytochemistry 1997, 45, 59-66. [CrossRef]

67. Rask, L.; Andréasson, E.; Ekbom, B.; Eriksson, S.; Pontoppidan, B.; Meijer, J. Myrosinase: Gene family evolution and herbivore defense in Brassicaceae. Plant Mol. Biol. 2000, 42, 93-114. [CrossRef] [PubMed]

68. Uda, Y.; Kurata, T.; Arakawa, N. Effects of $\mathrm{pH}$ and ferrous ion on the degradation of glucosinolates by myrosinase. Agric. Biol. Chem. 1986, 50, 2735-2740. [CrossRef]

69. Lambrix, V.; Reichelt, M.; Mitchell-Olds, T.; Kliebenstein, D.J.; Gershenzon, J. The arabidopsis epithiospecifier protein promotes the hydrolysis of glucosinolates to nitriles and influences trichoplusia ni herbivory. Plant Cell 2001, 13, 2793-2807. [CrossRef] [PubMed]

70. Oliviero, T.; Verkerk, R.; Dekker, M. Isothiocyanates from Brassica vegetables-effects of processing, cooking, mastication, and digestion. Mol. Nutr. Food Res. 2018, 62, e1701069. [CrossRef]

71. Wittstock, U.; Burow, M. Tipping the scales-Specifier proteins in glucosinolate hydrolysis. IUBMB Life 2007, 59, 744-751. [CrossRef]

72. Hasapis, X.; MacLeod, A.J. Benzylglucosinolate degradation in heat-treated Lepidium sativum seeds and detection of a thiocyanateforming factor. Phytochemistry 1982, 21, 1009-1013. [CrossRef] 
73. Kim, J.H.; Lee, B.W.; Schroeder, F.; Jander, G. Identification of indole glucosinolate breakdown products with antifeedant effects on Myzus persicae (green peach aphid). Plant J. 2008, 54, 1015-1026. [CrossRef]

74. Burow, M.; Zhang, Z.-Y.; Ober, J.A.; Lambrix, V.M.; Wittstock, U.; Gershenzon, J.; Kliebenstein, D.J. ESP and ESM1 mediate indol-3-acetonitrile production from indol-3-ylmethyl glucosinolate in arabidopsis. Phytochemistry 2008, 69, 663-671. [CrossRef]

75. Agerbirk, N.; Olsen, C.E.; Sørensen, H. Initial and final products, nitriles, and ascorbigens produced in myrosinase-catalyzed hydrolysis of indole glucosinolates. J. Agric. Food Chem. 1998, 46, 1563-1571. [CrossRef]

76. Liang, H.; Yuan, Q.; Dong, H.; Liu, Y. Determination of sulforaphane in broccoli and cabbage by high-performance liquid chromatography. J. Food Compos. Anal. 2006, 19, 473-476. [CrossRef]

77. Villatoro-Pulido, M.; Priego-Capote, F.; Álvarez-Sánchez, B.; Saha, S.; Philo, M.; Obregón-Cano, S.; De Haro-Bailón, A.; Font, R.; Del Río-Celestino, M. An approach to the phytochemical profiling of rocket [Eruca sativa (Mill.) Thell]. J. Sci. Food Agric. 2013, 93, 3809-3819. [CrossRef] [PubMed]

78. Sangthong, S.; Weerapreeyakul, N. Simultaneous quantification of sulforaphene and sulforaphane by reverse phase HPLC and their content in Raphanus sativus L. var. caudatus Alef extracts. Food Chem. 2016, 201, 139-144. [CrossRef] [PubMed]

79. Wang, N.; Shen, L.; Qiu, S.; Wang, X.; Wang, K.; Hao, J.; Xu, M. Analysis of the isothiocyanates present in three Chinese Brassica vegetable seeds and their potential anticancer bioactivities. Eur. Food Res. Technol. 2010, 231, 951-958. [CrossRef]

80. Lv, C.; Zhang, Y.; Zou, L.; Sun, J.; Song, X.; Mao, J.; Wu, Y. Simultaneous hydrolysis and extraction increased erucin yield from broccoli seeds. ACS Omega 2021, 6, 6385-6392. [CrossRef] [PubMed]

81. Sosińska, E.; Obiedziński, M.W. Effect of processing on the content of glucobrassicin and its degradation products in broccoli and cauliflower. Food Control. 2011, 22, 1348-1356. [CrossRef]

82. Vieites-Outes, C.; López-Hernández, J.; Lage-Yusty, M.A. Modification of glucosinolates in turnip greens (Brassica rapa subsp. rapa L.) subjected to culinary heat processes. CyTA J. Food 2016, 14, 536-540. [CrossRef]

83. Rodrigues, L.; Silva, I.; Poejo, J.; Serra, A.T.; Matias, A.A.; Simplício, A.L.; Bronze, M.; Duarte, C.M.M. Recovery of antioxidant and antiproliferative compounds from watercress using pressurized fluid extraction. RSC Adv. 2016, 6, 30905-30918. [CrossRef]

84. Devys, M.; Barbier, M. Indole-3-carboxaldehyde in the cabbage Brassica oleracea: A systematic determination. Phytochemistry 1991, 30, 389-391. [CrossRef]

85. Vallejo, F.; Garcia-Viguera, C.; Tomas-Barberan, F. Changes in broccoli (Brassica oleracea L. Var. italica) health-promoting compounds with inflorescence development. J. Agric. Food Chem. 2003, 51, 3776-3782. [CrossRef]

86. Kupke, F.; Herz, C.; Hanschen, F.S.; Platz, S.; Odongo, G.A.; Helmig, S.; Rodríguez, M.M.B.; Schreiner, M.; Rohn, S.; Lamy, E. Cytotoxic and genotoxic potential of food-borne nitriles in a liver In Vitro model. Sci. Rep. 2016, 6, 37631. [CrossRef] [PubMed]

87. Kapusta-Duch, J.; Kusznierewicz, B.; Leszczyńska, T.; Borczak, B. Effect of cooking on the contents of glucosinolates and their degradation products in selected Brassica vegetables. J. Funct. Foods 2016, 23, 412-422. [CrossRef]

88. Eib, S.; Schneider, D.J.; Hensel, O.; Seuß-Baum, I. Relationship between mustard pungency and allyl-isothiocyanate content: A comparison of sensory and chemical evaluations. J. Food Sci. 2020, 85, 2728-2736. [CrossRef]

89. Baenas, N.; Marhuenda, J.; García-Viguera, C.; Zafrilla, P.; Moreno, D.A. Influence of cooking methods on glucosinolates and isothiocyanates content in novel cruciferous foods. Foods 2019, 8, 257. [CrossRef]

90. Fusari, C.M.; Ramirez, D.A.; Camargo, A.B. Simplified analytical methodology for glucosinolate hydrolysis products: A miniaturized extraction technique and multivariate optimization. Anal. Methods 2018, 11, 309-316. [CrossRef]

91. Matthäus, B.; Fiebig, H.-J. Simultaneous determination of isothiocyanates, indoles, and oxazolidinethiones in myrosinase digests of rapeseeds and rapeseed meal by HPLC. J. Agric. Food Chem. 1996, 44, 3894-3899. [CrossRef]

92. Li, Z.; Wei, X.; Liu, Y.; Fang, Z.; Yang, L.; Zhuang, M.; Zhang, Y.; Lv, H. Development of a simple method for determination of anti-cancer component of indole-3-carbinol in cabbage and broccoli. J. Food Nutr. Res. 2017, 5, 642-648. [CrossRef]

93. Han, D.; Row, K.H. Separation and purification of sulforaphane from broccoli by solid phase extraction. Int. J. Mol. Sci. 2011, 12, 1854-1861. [CrossRef]

94. Andini, S.; Araya-Cloutier, C.; Sanders, M.; Vincken, J.-P. Simultaneous analysis of glucosinolates and isothiocyanates by reversedphase ultra-high-performance liquid chromatography-electron spray ionization-tandem mass spectrometry. J. Agric. Food Chem. 2020, 68, 3121-3131. [CrossRef]

95. Campas-Baypoli, O.N.; Sánchez-Machado, D.I.; Bueno-Solano, C.; Ramírez-Wong, B.; López-Cervantes, J. HPLC method validation for measurement of sulforaphane level in broccoli by-products. Biomed. Chromatogr. 2009, 24, 387-392. [CrossRef]

96. Ciska, E.; Honke, J. Effect of the pasteurization process on the contents of ascorbigen, indole-3-carbinol, indole-3-acetonitrile, and 3,3'-diindolylmethane in fermented cabbage. J. Agric. Food Chem. 2012, 60, 3645-3649. [CrossRef]

97. González, F.; Quintero, J.; Del Río, R.; Mahn, A. Optimization of an extraction process to obtain a food-grade sulforaphane-rich extract from broccoli (Brassica oleracea var. italica). Molecules 2021, 26, 4042. [CrossRef] [PubMed]

98. Liang, H.; Li, C.; Yuan, Q.; Vriesekoop, F. Separation and purification of sulforaphane from broccoli seeds by solid phase extraction and preparative high-performance liquid chromatography. J. Agric. Food Chem. 2007, 55, 8047-8053. [CrossRef]

99. Ciska, E.; Honke, J.; Drabińska, N. Changes in glucosinolates and their breakdown products during the fermentation of cabbage and prolonged storage of sauerkraut: Focus on sauerkraut juice. Food Chem. 2021, 365, 130498. [CrossRef]

100. Li, J.; Xie, B.; Yan, S.; Li, H.; Wang, Q. Extraction and determination of 4-methylthio-3-butenyl isothiocyanate in Chinese radish (Raphanus sativus L.) roots. LWT 2015, 60, 1080-1087. [CrossRef] 
101. Han, Z.; Li, H.; Yu, X.-C.; Sun, D.-W. Effects of low temperature cooking on the retention of 4-(methylthio)-3-butenyl isothiocyanate (MTBITC) of Chinese white radish (Raphanus sativus L.). Food Bioprocess Technol. 2016, 9, 1640-1647. [CrossRef]

102. Wang, J.; Yu, H.; Zhao, Z.; Sheng, X.; Shen, Y.; Gu, H. Natural variation of glucosinolates and their breakdown products in broccoli (Brassica oleracea var. italica) Seeds. J. Agric. Food Chem. 2019, 67, 12528-12537. [CrossRef]

103. Kroener, E.-M.; Buettner, A. Sensory-analytical comparison of the aroma of different horseradish varieties (Armoracia rusticana). Front. Chem. 2018, 6, 149. [CrossRef] [PubMed]

104. Sharma, A.; Rai, P.; Prasad, S. GC-MS detection and determination of major volatile compounds in Brassica juncea L. leaves and seeds. Microchem. J. 2018, 138, 488-493. [CrossRef]

105. Klopsch, R.; Witzel, K.; Artemyeva, A.; Ruppel, S.; Hanschen, F.S. Genotypic variation of glucosinolates and their breakdown products in leaves of Brassica rapa. J. Agric. Food Chem. 2018, 66, 5481-5490. [CrossRef]

106. Zeng, W.; Tao, H.; Li, Y.; Wang, J.; Xia, C.; Li, S.; Wang, M.; Wang, Q.; Miao, H. The flavor of Chinese kale sprouts is affected by genotypic variation of glucosinolates and their breakdown products. Food Chem. 2021, 359, 129824. [CrossRef]

107. Marchioni, I.; Martinelli, M.; Ascrizzi, R.; Gabbrielli, C.; Flamini, G.; Pistelli, L.; Pistelli, L. Small functional foods: Comparative phytochemical and nutritional analyses of five microgreens of the Brassicaceae family. Foods 2021, 10, 427. [CrossRef]

108. Blažević, I.; Mastelić, J. Glucosinolate degradation products and other bound and free volatiles in the leaves and roots of radish (Raphanus sativus L.). Food Chem. 2009, 113, 96-102. [CrossRef]

109. Daryaei, H.; Yousef, A.E.; Balasubramaniam, V.M. Microbiological aspects of high-pressure processing of food: Inactivation of microbial vegetative cells and spores. In High Pressure Processing of Food: Principles, Technology and Applications; Balasubramaniam, V.M., Barbosa-Cánovas, G.V., Lelieveld, H.L.M., Eds.; Springer: New York, NY, USA, 2016; pp. 271-294, ISBN 978-1-4939-3234-4.

110. Ke, Y.-Y.; Shyu, Y.-T.; Wu, S.-J. Evaluating the anti-inflammatory and antioxidant effects of broccoli treated with high hydrostatic pressure in cell models. Foods 2021, 10, 167. [CrossRef] [PubMed]

111. Westphal, A.; Riedl, K.; Cooperstone, J.L.; Kamat, S.; Balasubramaniam, V.M.; Schwartz, S.J.; Böhm, V. High-pressure processing of broccoli sprouts: Influence on bioactivation of glucosinolates to isothiocyanates. J. Agric. Food Chem. 2017, 65, 8578-8585. [CrossRef] [PubMed]

112. Koo, S.Y.; Cha, K.H.; Song, D.-G.; Chung, D.; Lee, D.-U.; Pan, C.-H. Amplification of allyl isothiocyanate in red cabbage using high hydrostatic pressure treatment. J. Med. Plants Res. 2011, 5, 3819-3822. [CrossRef]

113. Amofa-Diatuo, T.; Anang, D.M.; Barba, F.J.; Tiwari, B.K. Development of new apple beverages rich in isothiocyanates by using extracts obtained from ultrasound-treated cauliflower by-products: Evaluation of physical properties and consumer acceptance. J. Food Compos. Anal. 2017, 61, 73-81. [CrossRef]

114. Barba, F.J.; Boussetta, N.; Vorobiev, E. Emerging technologies for the recovery of isothiocyanates, protein and phenolic compounds from rapeseed and rapeseed press-cake: Effect of high voltage electrical discharges. Innov. Food Sci. Emerg. Technol. 2015, 31, 67-72. [CrossRef]

115. Tanongkankit, Y.; Sablani, S.S.; Chiewchan, N.; Devahastin, S. Microwave-assisted extraction of sulforaphane from white cabbages: Effects of extraction condition, solvent and sample pretreatment. J. Food Eng. 2013, 117, 151-157. [CrossRef]

116. Li, L.; Lee, W.; Lee, W.J.; Auh, J.H.; Kim, S.S.; Yoon, J. Extraction of allyl isothiocyanate from wasabi (Wasabia japonica Matsum) using supercritical carbon dioxide. Food Sci. Biotechnol. 2010, 19, 405-410. [CrossRef]

117. Wu, H.; Zhang, G.-A.; Zeng, S.; Lin, K.-C. Extraction of allyl isothiocyanate from horseradish (Armoracia rusticana) and its fumigant insecticidal activity on four stored-product pests of paddy. Pest Manag. Sci. 2009, 65, 1003-1008. [CrossRef] [PubMed]

118. Rafińska, K.; Pomastowski, P.; Rudnicka, J.; Krakowska, A.; Maruśka, A.; Narkute, M.; Buszewski, B. Effect of solvent and extraction technique on composition and biological activity of Lepidium sativum extracts. Food Chem. 2019, 289, 16-25. [CrossRef]

119. Boussetta, N.; Lesaint, O.; Vorobiev, E. A study of mechanisms involved during the extraction of polyphenols from grape seeds by pulsed electrical discharges. Innov. Food Sci. Emerg. Technol. 2013, 19, 124-132. [CrossRef]

120. Li, Z.; Fan, Y.; Xi, J. Recent advances in high voltage electric discharge extraction of bioactive ingredients from plant materials. Food Chem. 2019, 277, 246-260. [CrossRef]

121. Tabasso, S.; Carnaroglio, D.; Gaudino, E.C.; Cravotto, G. Microwave, ultrasound and ball mill procedures for bio-waste valorisation. Green Chem. 2014, 17, 684-693. [CrossRef]

122. Bosiljkov, T.; Dujmić, F.; Bubalo, M.C.; Hribar, J.; Vidrih, R.; Brnčić, M.; Zlatic, E.; Redovniković, I.R.; Jokić, S. Natural deep eutectic solvents and ultrasound-assisted extraction: Green approaches for extraction of wine lees anthocyanins. Food Bioprod. Process. 2017, 102, 195-203. [CrossRef]

123. Bagade, S.B.; Patil, M. Recent advances in microwave assisted extraction of bioactive compounds from complex herbal samples: A review. Crit. Rev. Anal. Chem. 2021, 51, 138-149. [CrossRef] [PubMed]

124. Yang, Y.; Hu, B. Bio-based chemicals from biorefining: Lipid and wax conversion and utilization. In Advances in Biorefineries; Waldron, K., Ed.; Woodhead Publishing: Cambridge, UK, 2014; pp. 693-720.

125. Jaiswal, A.K.; Abu-Ghannam, N. Fermentation-assisted extraction of isothiocyanates from Brassica vegetable using box-behnken experimental design. Foods 2016, 5, 75. [CrossRef] [PubMed]

126. Yuan, Y.; Chen, H.; Han, Y.; Qiao, F.; Yan, H. Analysis of anticancer compound, indole-3-carbinol, in broccoli using a new ultrasound-assisted dispersive-filter extraction method based on poly (deep eutectic solvent)-graphene oxide nanocomposite. $J$. Pharm. Anal 2021, in press. [CrossRef] 
127. Coscueta, E.; Reis, C.; Pintado, M. Phenylethyl isothiocyanate extracted from watercress by-products with aqueous micellar systems: Development and optimisation. Antioxidants 2020, 9, 698. [CrossRef]

128. Passos, M.L.C.; Sarraguça, M.C.; Saraiva, M.L.M.F.S.; Prasada Rao, T.; Biju, V.M. Spectrophotometry l organic compounds. In Encyclopedia of Analytical Science, 3rd ed.; Worsfold, P., Poole, C., Townshend, A., Miró, M., Eds.; Academic Press: Oxford, UK, 2019; pp. 350-359, ISBN 9780081019849.

129. Zhang, Y. The 1,2-benzenedithiole-based cyclocondensation assay: A valuable tool for the measurement of chemopreventive isothiocyanates. Crit. Rev. Food Sci. Nutr. 2012, 52, 525-532. [CrossRef] [PubMed]

130. Jiao, D.; Yu, M.C.; Hankin, J.H.; Low, S.-H.; Chung, F.-L. Total isothiocyanate contents in cooked vegetables frequently consumed in Singapore. J. Agric. Food Chem. 1998, 46, 1055-1058. [CrossRef]

131. Sun, J.; Chen, P. Quantification of total glucosinolates and isothiocyanates for common Brassicaceous vegetables consumed in the US market using cyclocondensation and thiocyanate ion measurement methods. J. Anal. Test. 2019, 3, 313-321. [CrossRef]

132. Choi, M.M.; Shuang, S.; Lai, H.; Cheng, S.; Cheng, R.C.; Cheung, B.K.; Lee, A.W. Gas chromatography-mass spectrometric determination of total isothiocyanates in Chinese medicinal herbs. Anal. Chim. Acta 2004, 516, 155-163. [CrossRef]

133. Mirabella, F.M. Internal Reflection Spectroscopy: Theory and Applications; Mirabella, F.M., Ed.; CRC Press: Boca Raton, FL, USA, 2020; ISBN 978-1-00-306694-1.

134. Clapp, R.C.; Long, L.; Dateo, G.P.; Bissett, F.H.; Hasselstrom, T. The volatile isothiocyanates in fresh cabbage ${ }^{1}$. J. Am. Chem. Soc. 1959, 81, 6278-6281. [CrossRef]

135. Daxenbichler, M.E.; VanEtten, C.H.; Brown, F.S.; Jones, Q. Seed meal constituents, oxazolidinethiones and volatile isothiocyanates in enzyme-treated seed meals from 65 species of Cruciferae. J. Agric. Food Chem. 1964, 12, 127-130. [CrossRef]

136. Kjaer, A.; Rubinstein, K. Paper chromatography of thioureas. Nat. Cell Biol. 1953, 171, 840-841. [CrossRef]

137. Kore, A.M.; Spencer, G.F.; Wallig, M.A. Purification of the.omega.-(methylsulfinyl) alkyl glucosinolate hydrolysis products: 1-isothiocyanato-3-(methylsulfinyl) propane, 1-isothiocyanato-4-(methylsulfinyl) butane, 4-(methylsulfinyl) butanenitrile, and 5-(methylsulfinyl) pentanenitrile from broccoli and Lesquerella fendleri. J. Agric. Food Chem. 1993, 41, 89-95. [CrossRef]

138. Guo, R.; Yuan, G.; Wang, Q. Effect of sucrose and mannitol on the accumulation of health-promoting compounds and the activity of metabolic enzymes in broccoli sprouts. Sci. Hortic. 2011, 128, 159-165. [CrossRef]

139. Guo, R.-F.; Yuan, G.-F.; Wang, Q.-M. Effect of $\mathrm{NaCl}$ treatments on glucosinolate metabolism in broccoli sprouts. J. Zhejiang Univ. Sci. B 2013, 14, 124-131. [CrossRef]

140. Matusheski, N.; Wallig, M.A.; Juvik, J.A.; Klein, B.P.; Kushad, M.M.; Jeffery, E. Preparative HPLC method for the purification of sulforaphane and sulforaphane nitrile from Brassica oleracea. J. Agric. Food Chem. 2001, 49, 1867-1872. [CrossRef]

141. Jin, Y.; Wang, M.; Rosen, R.T.; Ho, C.-T. Thermal degradation of sulforaphane in aqueous solution. J. Agric. Food Chem. 1999, 47, 3121-3123. [CrossRef]

142. Chen, C.-W.; Ho, C.-T. Thermal degradation of allyl isothiocyanate in aqueous solution. J. Agric. Food Chem. 1998, 46, $220-223$. [CrossRef]

143. Pilipczuk, T.; Dawidowska, N.; Kusznierewicz, B.; Namiesnik, J.; Bartoszek, A. Simultaneous determination of indolic compounds in plant extracts by solid-phase extraction and high-performance liquid chromatography with UV and fluorescence detection. Food Anal. Methods 2015, 8, 2169-2177. [CrossRef]

144. Nakagawa, K.; Umeda, T.; Higuchi, O.; Tsuduki, T.; Miyazawa, T. Evaporative light-scattering analysis of sulforaphane in broccoli samples: Quality of broccoli products regarding sulforaphane contents. J. Agric. Food Chem. 2006, 54, 2479-2483. [CrossRef]

145. Wang, T.; Wang, Q.; Li, P.; Yang, H. High-speed countercurrent chromatography-based method for simultaneous recovery and separation of natural products from deep eutectic solvent extracts. ACS Sustain. Chem. Eng. 2020, 8, 2073-2080. [CrossRef]

146. Khan, B.M.; Liu, Y. High speed counter current chromatography: Overview of solvent-system and elution-mode. J. Liq. Chromatogr. Relat. Technol. 2018, 41, 629-636. [CrossRef]

147. Liang, H.; Li, C.; Yuan, Q.; Vriesekoop, F. Application of high-speed countercurrent chromatography for the isolation of sulforaphane from broccoli seed meal. J. Agric. Food Chem. 2008, 56, 7746-7749. [CrossRef] [PubMed]

148. Kuang, P.; Song, D.; Yuan, Q.; Lv, X.; Zhao, D.; Liang, H. Preparative separation and purification of sulforaphene from radish seeds by high-speed countercurrent chromatography. Food Chem. 2013, 136, 309-315. [CrossRef] [PubMed]

149. Chew, S.C.; Yang, L. Chromatography: Supercritical fluid chromatography. In Encyclopedia of Food and Health; Caballero, B., Finglas, P.M., Toldrá, F., Eds.; Academic Press: Oxford, UK, 2016; pp. 407-415, ISBN 978-0-12-384953-3.

150. Buskov, S.; Hansen, L.B.; Olsen, C.E.; Sørensen, J.C.; Sørensen, H.; Sørensen, S. Determination of ascorbigens in autolysates of various Brassica species using supercritical fluid chromatography. J. Agric. Food Chem. 2000, 48, 2693-2701. [CrossRef] [PubMed]

151. Buskov, S.; Hasselstrøm, J.; Olsen, C.E.; Sørensen, H.; Sørensen, J.C.; Sørensen, S. Supercritical fluid chromatography as a method of analysis for the determination of 4-hydroxybenzylglucosinolate degradation products. J. Biochem. Biophys. Methods 2000, 43, 157-174. [CrossRef]

152. Van Eylen, D.; Bellostas, N.; Strobel, B.W.; Oey, I.; Hendrickx, M.E.; Van Loey, A.; Sørensen, H.; Sørensen, J. Influence of pressure/temperature treatments on glucosinolate conversion in broccoli (Brassica oleraceae L. cv italica) heads. Food Chem. 2009, 112, 646-653. [CrossRef]

153. Pilipczuk, T.; Kusznierewicz, B.; Chmiel, T.; Przychodzeń, W.; Bartoszek, A. Simultaneous determination of individual isothiocyanates in plant samples by HPLC-DAD-MS following SPE and derivatization with N -acetyl- 1 -cysteine. Food Chem. 2017, 214, 587-596. [CrossRef] [PubMed] 
154. Ghawi, S.K.; Methven, L.; Niranjan, K. The potential to intensify sulforaphane formation in cooked broccoli (Brassica oleracea var. italica) using mustard seeds (Sinapis alba). Food Chem. 2013, 138, 1734-1741. [CrossRef]

155. Shen, L.; Su, G.; Wang, X.; Du, Q.; Wang, K. Endogenous and exogenous enzymolysis of vegetable-sourced glucosinolates and influencing factors. Food Chem. 2009, 119, 987-994. [CrossRef]

156. Naser, S.A.; Amiri-Besheli, B.; Sharifi-Mehr, S. The isolation and determination of sulforaphane from broccoli tissues by reverse phase-high performance liquid chromatography. J. Chin. Chem. Soc. 2011, 58, 906-910. [CrossRef]

157. Raffo, A.; Masci, M.; Moneta, E.; Nicoli, S.; del Pulgar, J.S.; Paoletti, F. Characterization of volatiles and identification of odor-active compounds of rocket leaves. Food Chem. 2018, 240, 1161-1170. [CrossRef]

158. Wieczorek, M.N.; Jeleń, H.H. Volatile compounds of selected raw and cooked Brassica vegetables. Molecules 2019, $24,391$. [CrossRef] [PubMed]

159. Wieczorek, M.N.; Majcher, M.; Jeleń, H. Comparison of three extraction techniques for the determination of volatile flavor components in broccoli. Foods 2020, 9, 398. [CrossRef] [PubMed]

160. Wieczorek, M.N.; Majcher, M.A.; Jeleń, H.H. Identification of aroma compounds in raw and cooked broccoli. Flavour Fragr. J. 2021, 36, 576-583. [CrossRef]

161. Wei, S.; Xiao, X.; Wei, L.; Li, L.; Li, G.; Liu, F.; Xie, J.; Yu, J.; Zhong, Y. Development and comprehensive HS-SPME/GC-MS analysis optimization, comparison, and evaluation of different cabbage cultivars (Brassica oleracea L. var. capitata L.) volatile components. Food Chem. 2021, 340, 128166. [CrossRef] [PubMed]

162. Gonda, S.; Kiss-Szikszai, A.; Szúcs, Z.; Nguyen, N.M.; Vasas, G. Myrosinase compatible simultaneous determination of glucosinolates and allyl isothiocyanate by capillary electrophoresis micellar electrokinetic chromatography (CE-MEKC). Phytochem. Anal. 2016, 27, 191-198. [CrossRef] [PubMed] 